\title{
EXISTENCE OF LATTICES IN KAC-MOODY GROUPS OVER FINITE FIELDS
}

\author{
LISA CARBONE \\ Department of Mathematics, Hill Center-Busch Campus, Rutgers, \\ The State University of New Jersey, 110 Frelinghuysen Rd Piscataway, \\ NJ 08854-8019, USA \\ carbonel@math.rutgers.edu \\ HOWARD GARLAND \\ Department of Mathematics, Yale University, \\ 10 Hillhouse Avenue, P.O. Box 208283, New Haven, CT 06520, USA \\ hgarland@snet.net
}

Received 6 April 2002

\begin{abstract}
Let $\mathfrak{g}$ be a Kac-Moody Lie algebra. We give an interpretation of Tits' associated group functor using representation theory of $\mathfrak{g}$ and we construct a locally compact "Kac-Moody group" $G$ over a finite field $k$. Using (twin) $B N$-pairs $(G, B, N)$ and $\left(G, B^{-}, N\right)$ for $G$ we show that if $k$ is "sufficiently large", then the subgroup $B^{-}$is a non-uniform lattice in $G$. We have also constructed an uncountably infinite family of both uniform and nonuniform lattices in rank 2 . We conjecture that these form uncountably many distinct conjugacy classes in $G$. The basic tool for the construction of non-uniform lattices in rank 2 is a spherical Tits system for $G$ which we also construct.
\end{abstract}

Keywords:

2000 Mathematics Subject Classification: Primary 20F32; Secondary 22F50

\section{Introduction}

Let $\mathfrak{g}$ be a Kac-Moody Lie algebra. In [29, 30], Tits associated to $\mathfrak{g}$ a group functor on the category of rings. In this work we give an interpretation of Tits' group functor using representation theory of $\mathfrak{g}$ (Secs. 5 and 6). We produce a "KacMoody group" $G$ that is a completion of Tits' "minimal" group in $[29,30]$. Let $G$ denote such a group over a finite field. The group $G$ is then locally compact and totally disconnected (Sec. 6).

Theorem 0.1. Let $G$ be a (complete) Kac-Moody group over a finite field $k$. The group $G$ has (twin) BN-pairs $(G, B, N)$ and $\left(G, B^{-}, N\right)$ where $B$ is (the completion of) the subgroup of $G$ generated by a "diagonal subgroup" $H$ and all positive real root groups, and $B^{-}$is the subgroup of $G$ generated by $H$ and all negative real root groups. 
The group $G$ admits a cocompact action on its corresponding Bruhat-Tits building, which is an affine or hyperbolic building in rank greater than 2, and a homogeneous tree in rank 2. Our aim is to determine if in analogy with Lie groups, $G$ contains lattices, that is discrete subgroups whose quotients carry a finite invariant measure. We describe a construction of non-uniform "arithmetic" lattices in Kac-Moody groups over sufficiently large finite fields using the Tits system for such groups (Theorem 8.1). B. Remy has obtained an equivalent result for the more general class of almost split Kac-Moody groups [25, 26].

Theorem 0.2. Let $G$ be a Kac-Moody group over a finite field $k$. If $k$ is "sufficiently large", then the subgroup $B^{-}$is a non-uniform lattice in $G$.

It follows that if $P^{-} \subseteq G$ is a proper standard parabolic subgroup for the $B N$-pair $\left(G, B^{-}, N\right),\left(B^{-} \subseteq P^{-} \subsetneq G\right)$, if the submatrix of $A$ corresponding to $P^{-}$ is positive-definite and if $k$ is "sufficiently large", then $P^{-}$is a non-uniform lattice in $G$ (Corollary 8.1).

We have also constructed an uncountably infinite family of both uniform and non-uniform lattices in the rank 2 Kac-Moody case (Theorems 11.1 and 15.1), by generalizing A. Lubotzky's construction of non-uniform lattices in $S L_{2}$ over a Laurent series field, using the Bruhat-Tits tree [18].

Theorem 0.3. If $G$ has rank 2 , then $G$ contains uncountably infinite families of both uniform and non-uniform lattices.

The rank of $G$ is defined to be $l=|I|$ as in Sec. 1. The basic tool for the construction of non-uniform lattices in rank 2 is a (new) spherical Tits system for the Kac-Moody group in rank 2 (Sec. 14).

Theorem 0.4. Let $G$ be a Kac-Moody group over a finite field. If $G$ has rank 2, then $G$ has a spherical Tits-system.

The corresponding spherical building is 0-dimensional, and may be identified with $\partial X$, the boundary of the Bruhat-Tits tree $X$.

It remains to determine whether, as in the case of $S L_{2}$, we have constructed uncountably many distinct conjugacy classes of uniform (respectively non-uniform) lattices within the Kac-Moody group (Conjectures 12.1 and 16.1), while in contrast we show that these uniform (respectively non-uniform) lattices are all conjugate in the automorphism group of the Bruhat-Tits tree (see Proposition 12.1, respectively Proposition 16.1).

Using the action of $G$ on its Bruhat-Tits tree in rank 2, we may deduce (Proposition 17.1):

Proposition 0.1. If $G$ has rank $2, G$ does not have property $T$.

In contrast to this, in the higher rank case, results of Dymara and Januszkiewicz $[10,11]$ imply that certain Kac-Moody groups do have property $T$. Hence, the 
lattices that we construct in these cases are finitely generated and have finite commutator quotients.

Regarding cohomology we have the following (Theorem 18.1 and Corollary 18.1):

Theorem 0.5. Let $l$ be the rank of $G, q=|k|$, let $\Gamma \leq G$ be a uniform lattice of $G$, and let $\rho: \Gamma \longrightarrow \operatorname{Aut}(V)$ be a unitary representation of $\Gamma$ in a complex Hilbert space $V$. If $l \geq 3$, and if $q$ is sufficiently large, then $H^{i}(\Gamma, \rho)=0,0<i<l-1$.

Corollary 0.1. If $G$ contains a uniform lattice, then $G$ has property $T$.

The paper is organized as follows. In Secs. 1-6, we describe the basic setting of Kac-Moody algebras and Kac-Moody groups. In Sec. 7, we consider some basic results about lattices in locally compact groups, and in Sec. 8 we describe our construction of non-uniform "arithmetic" lattices Kac-Moody groups over sufficiently large finite fields.

Sections 9-17 then focus on the case of rank 2. In Sec. 9 we outline the structure theory of the Bruhat-Tits tree in rank 2, and in Sec. 10 we describe A. Lubotzky's adaptation of the classical notion of a Schottky group of automorphisms of the upper half plane to the non-archimedean case, a notion central to our construction of lattices in rank 2. In Secs. 11 (uniform case) and 15 (non-uniform case) we generalize A. Lubotzky's construction of lattices in [18] to the Kac-Moody case, and in Secs. 12 (uniform case) and 16 (non-uniform case) we show how these constructions are flexible enough so as to admit uncountably infinite families of lattices in rank 2.

In Sec. 14 we introduce a spherical $B N$-pair for Kac-Moody groups of rank 2, and in Sec. 13, we outline some unexpected results regarding commensurability of uniform lattices in rank 2. In Secs. 17 and 18 we outline our results on the failure of property $T$ in rank 2, and cohomology of lattices. In an appendix we give explicit computations for symmetric, $2 \times 2$, generalized Cartan matrices.

\section{The Setting}

We let $A=\left(A_{i j}\right)_{i, j \in I}$ be a symmetrizable generalized Cartan matrix; that is, $I=$ $\{1,2, \ldots, l\}$ is a finite set, and $A=\left(A_{i j}\right)_{i, j \in I}$ satisfies:

(1) $A_{i j} \in \mathbb{Z}, i, j \in I$,

(2) $A_{i i}=2, i \in I$,

(3) $A_{i j} \leq 0$ if $i \neq j$,

(4) There exist positive rational numbers $q_{1}, \ldots, q_{l}$, such that the matrix $D A$ is symmetric, where $D=\operatorname{diag}\left(q_{1}, \ldots, q_{l}\right)$.

We remark that (4) implies:

$$
A_{i j}=0 \Longleftrightarrow A_{j i}=0 .
$$

By a proper submatrix of $A$, we mean a matrix of the form:

$$
A_{\theta}=\left(A_{i j}\right)_{i, j \in \theta}
$$

where $\theta$ is a proper subset of $\{1, \ldots, l\}$. 
We say that the matrix $A$ is indecomposable if there is no partition of the set $\{1, \ldots, l\}$ into two non-empty subsets so that $A_{i j}=0$ whenever $i$ belongs to the first subset, while $j$ belongs to the second.

An indecomposable symmetrizable generalized Cartan matrix $A$ is of:

(1) Classical type if, for $D$ as above, $D A$ is positive definite. In this case, $A$ is the Cartan matrix of a finite dimensional complex semi-simple Lie algebra.

(2) Affine type if for $D$ as above, the matrix $D A$ is positive semi-definite, but not positive definite.

(3) Hyperbolic type if $A$ is neither of classical nor affine type, but every proper, indecomposable submatrix is either of classical or of affine type.

If $A$ is of hyperbolic type, we say that $A$ is of compact hyperbolic type if every proper, indecomposable submatrix is of classical type.

Our conventions are consistent with those of $[2$, Chapt. 4$]$ which give a characterization hyperbolicity of $A$.

\section{Kac-Moody Algebras over $\mathbb{Q}$}

Following [16, Chapt. 1], we introduce the notion of a realization

$$
\left(\mathfrak{h}, \Pi, \Pi^{\nu}\right)
$$

of a symmetrizable generalized Cartan matrix $A$. Here $\mathfrak{h}=\mathfrak{h}_{\mathbb{Q}}$ is a vector space over $\mathbb{Q}$,

$$
\Pi=\left\{\alpha_{1}, \ldots, \alpha_{l}\right\} \subseteq \mathfrak{h}_{\mathbb{Q}}^{*}, \quad \text { the dual space of } \mathfrak{h}_{\mathbb{Q}},
$$

and the elements of $\Pi$ are called simple roots,

$$
\Pi^{\nu}=\left\{\alpha_{1}^{\nu}, \ldots, \alpha_{l}^{\nu}\right\} \subseteq \mathfrak{h}_{\mathbb{Q}},
$$

and the elements of $\Pi^{\nu}$ are called simple coroots. The data $\left(\mathfrak{h}_{\mathbb{Q}}, \Pi, \Pi^{\nu}\right)$ is subject to the conditions

(1) The sets $\Pi$ and $\Pi^{\nu}$ are each linearly independant,

(2) $\alpha_{j}\left(\alpha_{i}^{\nu}\right)=A_{i j}, i, j=1, \ldots, l$,

(3) If $n=\operatorname{rank}(A)$, then

$$
l-n=\operatorname{dim}\left(\mathfrak{h}_{\mathbb{Q}}\right)-l .
$$

Following [16, Chapt. 1], two realizations $\left(\mathfrak{h}, \Pi, \Pi^{\nu}\right)$ and $\left(\mathfrak{h}_{1}, \Pi_{1}, \Pi_{1}^{\nu}\right)$ of $A$ are said to be isomorphic if there is a vector space isomorphism $\phi: \mathfrak{h} \longrightarrow \mathfrak{h}_{1}$ such that $\phi\left(\Pi^{\nu}\right)=\Pi_{1}^{\nu}, \phi^{*}\left(\Pi_{1}\right)=\Pi$. In [16, Proposition 1.1] Kac proves that a symmetrizable generalized Cartan matrix, $A$, has a realization that is unique up to isomorphism.

Given a symmetrizable generalized Cartan matrix $A$, one can associate a KacMoody algebra $\mathfrak{g}_{\mathbb{Q}}=\mathfrak{g}_{\mathbb{Q}}(A)$, a Lie algebra over $\mathbb{Q}$, generated by $\mathfrak{h}=\mathfrak{h}_{\mathbb{Q}}$ and elements $\left(e_{i}\right)_{i \in I},\left(f_{i}\right)_{i \in I}$ subject to relations: 
(1) $[\mathfrak{h}, \mathfrak{h}]=0$,

(2) $\left[h, e_{i}\right]=\alpha_{i}(h) e_{i}, h \in \mathfrak{h}$,

(3) $\left[h, f_{i}\right]=-\alpha_{i}(h) f_{i}, h \in \mathfrak{h}$,

(4) $\left[e_{i}, f_{i}\right]=\alpha_{i}^{\nu}$,

(5) $\left[e_{i}, f_{j}\right]=0, i \neq j$,

(6) $\left(a d e_{i}\right)^{-A_{i j}+1}\left(e_{j}\right)=0, i \neq j$,

(7) $\left(a d f_{i}\right)^{-A_{i j}+1}\left(f_{j}\right)=0, i \neq j$.

If $A$ is of classical type, the algebra $\mathfrak{g}_{\mathbb{Q}}$ is finite dimensional. If $A$ is of affine type, $\mathfrak{g}_{\mathbb{Q}}$ is infinite dimensional but of polynomial growth. If $A$ is of hyperbolic type, $\mathfrak{g}_{\mathbb{Q}}$ is infinite dimensional and of exponential growth.

Let $\mathfrak{g}_{\mathbb{Q},+}$ be the Lie subalgebra of $\mathfrak{g}_{\mathbb{Q}}$ generated by $\left(e_{i}\right)_{i \in I}$ with defining relation $(6)$, and let $\mathfrak{g}_{\mathbb{Q},-}$ be the Lie subalgebra of $\mathfrak{g}_{\mathbb{Q}}$ generated by $\left(f_{i}\right)_{i \in I}$ with defining relation (7).

Theorem 2.1 ([29, p. 77]). The canonical map

$$
\mathfrak{g}_{\mathbb{Q},-} \oplus \mathfrak{h}_{\mathbb{Q}} \oplus \mathfrak{g}_{\mathbb{Q},+} \longrightarrow \mathfrak{g}_{\mathbb{Q}}
$$

is a bijection.

\section{Roots and Weyl Group}

The algebra $\mathfrak{g}_{\mathbb{Q}}$ decomposes into eigenspaces, called root spaces, under the simultaneous adjoint action of $\mathfrak{h}_{\mathbb{Q}}$. Here, a non-zero element $\alpha \in \mathfrak{h}_{\mathbb{Q}}^{*}$ is called a root of $\mathfrak{g}_{\mathbb{Q}}$ if there is a non-zero element $x \in \mathfrak{g}_{\mathbb{Q}}$, such that

$$
[h, x]=\alpha(h) x, \quad h \in \mathfrak{h} .
$$

We set $\Delta=\Delta(A)$ to be the set of all roots of $\mathfrak{g}_{\mathbb{Q}}$. Then $\mathfrak{g}_{\mathbb{Q}}$ has a direct sum decomposition

$$
\mathfrak{g}_{\mathbb{Q}}=\mathfrak{h}_{\mathbb{Q}} \oplus \bigsqcup_{\alpha \in \Delta(A)} \mathfrak{g}_{\mathbb{Q}}^{\alpha},
$$

where

$$
\mathfrak{g}_{\mathbb{Q}}^{\alpha}=\left\{x \in \mathfrak{g}_{\mathbb{Q}} \mid[h, x]=\alpha(h) x, h \in \mathfrak{h}_{\mathbb{Q}}\right\} .
$$

Each $\mathfrak{g}_{\mathbb{Q}}^{\alpha}$ is finite dimensional. In particular,

$$
\pm \alpha_{i} \in \Delta(A), \quad i \in I
$$

and $\mathfrak{g}_{\mathbb{Q}}^{\alpha_{i}}=\mathbb{Q} e_{i}, \mathfrak{g}_{\mathbb{Q}}^{-\alpha_{i}}=\mathbb{Q} f_{i}, i=1, \ldots, l$. The elements $\alpha_{1}, \ldots, \alpha_{l}$ are the simple roots. Every root $\alpha \in \Delta(A)$ has a unique expression

$$
\alpha=\sum_{i=1}^{l} k_{i} \alpha_{i},
$$


where the $k_{i}$ are either all in $\mathbb{Z}_{\geq 0}$, in which case we say that $\alpha$ is positive, or the $k_{i}$ are all in $\mathbb{Z}_{\leq 0}$, in which case we say that $\alpha$ is negative. We set

$$
\Delta^{ \pm}=\Delta^{ \pm}(A)
$$

equal to the set of \pm roots. Then

$$
\Delta=\Delta^{+} \cup \Delta^{-} \quad \text { (disjoint union). }
$$

We set

$$
\mathfrak{u}_{ \pm}:=\mathfrak{u}_{ \pm}(A)=\bigsqcup_{\alpha \in \Delta^{ \pm}} \mathfrak{g}_{\mathbb{Q}}^{\alpha} .
$$

Then $\mathfrak{u}_{ \pm}$is a subalgebra of $\mathfrak{g}_{\mathbb{Q}}$, and in fact equals $\mathfrak{g}_{\mathbb{Q}, \pm}$. Moreover $\mathfrak{g}_{\mathbb{Q}}$ admits an invariant, symmetric bilinear form $\sigma($,$) , and \sigma$ induces a non-degenerate pairing between $\mathfrak{g}_{\mathbb{Q}}^{\alpha}$ and $\mathfrak{g}_{\mathbb{Q}}^{-\alpha}$, for $\alpha \in \Delta(A)$. We have the Weyl reflections

$$
w_{i}: \mathfrak{h} \longrightarrow \mathfrak{h}, \quad i=1, \ldots, l,
$$

where

$$
w_{i}(h)=h-\alpha_{i}(h) \alpha_{i}^{\nu}, \quad h \in \mathfrak{h} .
$$

The $w_{i}$ generate a subgroup $W=W(A) \subseteq \operatorname{Aut}(\mathfrak{h})$, called the Weyl group of $\mathfrak{g}_{\mathbb{Q}}$, and $\left.\sigma\right|_{\mathfrak{h}}$ is $W$-invariant. For $i, j \in I$, we set

$$
c_{i i}:=1
$$

and for $i \neq j$,

$$
c_{i j}:=2,3,4,6, \quad \text { or } \infty
$$

according as

$$
A_{i j} A_{j i}=0,1,2,3, \quad \text { or } \geq 4 .
$$

Then $W=W(A)$ is the group with presentation (see [29, p. 81])

$$
\left.W=\left\langle m_{i}\right| i \in I,\left(m_{i} m_{j}\right)^{c_{i j}}=1, \quad \text { if } c_{i j} \neq \infty\right\rangle .
$$

A root $\alpha \in \Delta$ is called a real root (or a Weyl root) if there exists $w \in W$ such that $w(\alpha)$ is a simple root. A root $\alpha$ which is not real is called imaginary. We denote by $\Phi$ the Weyl roots, $\Phi_{I}$ the imaginary roots, $\Phi^{ \pm}$the positive and negative Weyl roots, and $\Phi_{I}^{ \pm}$the positive and negative imaginary roots. Then

$$
\begin{gathered}
\Delta=\Phi \sqcup \Phi_{I}, \\
\Phi=\Phi^{+} \sqcup \Phi^{-}, \\
\Phi_{I}=\Phi_{I}^{+} \sqcup \Phi_{I}^{-},
\end{gathered}
$$

and

$$
\Phi=W(\Pi)
$$




\section{A $\mathbb{Z}$-Form of the Universal Enveloping Algebra of $\mathfrak{g}_{\mathbb{Q}}$}

Let $\mathcal{U}_{\mathbb{Q}}, \mathcal{U}_{\mathbb{Q},+}$ and $\mathcal{U}_{\mathbb{Q},-}$ be the universal enveloping algebras of $\mathfrak{g}_{\mathbb{Q}}, \mathfrak{g}_{\mathbb{Q},+}$ and $\mathfrak{g}_{\mathbb{Q},-}$ respectively. The following result follows from the Birkhoff-Witt theorem.

Theorem $4.1([\mathbf{2 9}, \mathbf{p . 7 7}])$. Let $\mathcal{S}\left(\mathfrak{h}_{\mathbb{Q}}\right)$ be the symmetric algebra of $\mathfrak{h}_{\mathbb{Q}}$. The canonical map

$$
\mathcal{U}_{\mathbb{Q},-} \otimes \mathcal{S}\left(\mathfrak{h}_{\mathbb{Q}}\right) \otimes \mathcal{U}_{\mathbb{Q},+} \longrightarrow \mathcal{U}_{\mathbb{Q}}
$$

is a bijection.

For any associative algebra $A$, let $x \in A$ and let $m \in \mathbb{Z}_{\geq 0}$. We set

$$
x^{(m)}:=\frac{x^{m}}{m !},
$$

and

$$
\left(\begin{array}{c}
x \\
m
\end{array}\right):=\frac{x(x-1)(x-2) \cdots(x-m+1)}{m !} .
$$

We choose $\Lambda \subseteq \mathfrak{h}^{*}$ to be the linear span of $\alpha_{i}$, for $i \in I$, and $\Lambda^{\prime} \subseteq \mathfrak{h}$ to be the linear span of $h_{i}$, (where $\left.h_{i}=\alpha_{i}^{\nu}\right)$, for $i \in I$. Let

$\mathcal{U}_{\mathbb{Z},+} \subseteq \mathcal{U}_{\mathbb{Q},+}$ be the $\mathbb{Z}$-subalgebra generated by $e_{i}^{(m)}$ for $i \in I$ and $m \geq 0$,

$\mathcal{U}_{\mathbb{Z},-} \subseteq \mathcal{U}_{\mathbb{Q},-}$ be the $\mathbb{Z}$-subalgebra generated by $f_{i}^{(m)}$ for $i \in I$ and $m \geq 0$,

$\mathcal{U}_{\mathbb{Z}, 0} \subseteq \mathcal{S}\left(\mathfrak{h}_{\mathbb{Q}}\right)$ be the $\mathbb{Z}$-subalgebra generated by $\left(\begin{array}{l}\lambda^{\prime} \\ m\end{array}\right)$, for $\lambda^{\prime} \in \Lambda^{\prime}$ and $m \geq 0$, and let

$\mathcal{U}_{\mathbb{Z}} \subseteq \mathcal{U}_{\mathbb{Q}}$ be the $\mathbb{Z}$-subalgebra generated by $e_{i}^{(m)}, f_{i}^{(m)}$ for $i \in I$ and $\left(\begin{array}{l}\lambda^{\prime} \\ m\end{array}\right)$, for $\lambda^{\prime} \in \Lambda^{\prime}$ and $m \geq 0$. By a $\mathbb{Z}$-form of a $\mathbb{Q}$-algebra, $X_{\mathbb{Q}}$, we mean a subring $X$ of $X_{\mathbb{Q}}$ such that the canonical map

$$
X \otimes \mathbb{Q} \longrightarrow X_{\mathbb{Q}}
$$

is bijective. Using the standard grading of $\mathcal{U}_{\mathbb{Q}}$ defined by

$$
\operatorname{deg}\left(e_{i}\right)=-\operatorname{deg}\left(f_{i}\right)=1,
$$

it follows that $\mathcal{U}_{\mathbb{Z},+}$ is a $\mathbb{Z}$-form of $\mathcal{U}_{\mathbb{Q},+}[29$, p. 78$]$.

We set

$$
\begin{aligned}
\mathfrak{g}_{\mathbb{Z}} & =\mathfrak{g}_{\mathbb{Q}} \cap \mathcal{U}_{\mathbb{Z}}, \\
\mathfrak{g}_{\mathbb{Z}, \pm} & =\mathfrak{g}_{\mathbb{Q}, \pm} \cap \mathcal{U}_{\mathbb{Z}} .
\end{aligned}
$$

The following theorem then implies that $\mathcal{U}_{\mathbb{Z}}$ is a $\mathbb{Z}$-form of $\mathcal{U}_{\mathbb{Q}}$.

Theorem 4.2 ([29, p. 78]). The product map

$$
\mathcal{U}_{\mathbb{Z},-} \otimes \mathcal{U}_{\mathbb{Z}, 0} \otimes \mathcal{U}_{\mathbb{Z},+} \longrightarrow \mathcal{U}_{\mathbb{Z}}
$$

and the sum map

$$
\mathfrak{g}_{\mathbb{Z},-} \oplus \Lambda^{\prime} \oplus \mathfrak{g}_{\mathbb{Z},+} \longrightarrow \mathfrak{g}_{\mathbb{Z}}
$$

are isomorphisms of modules. 
For a field $K$, we let

$$
\begin{aligned}
\mathcal{U}_{K} & =\mathcal{U}_{\mathbb{Z}} \otimes K \\
\mathcal{U}_{K, 0} & =\mathcal{U}_{\mathbb{Z}, 0} \otimes K \\
\mathcal{U}_{K, \pm} & =\mathcal{U}_{\mathbb{Z}, \pm} \otimes K \\
\mathfrak{g}_{K} & =\mathfrak{g}_{\mathbb{Z}} \otimes K \\
\mathfrak{g}_{K, \pm} & =\mathfrak{g}_{\mathbb{Z}, \pm} \otimes K
\end{aligned}
$$

\section{Notation}

We will construct Kac-Moody groups by means of representation theory. The groups obtained in this way (Sec. 6) depend on the chosen representation. Before completion, the groups constructed (in Sec. 6) are homomorphic images of the "minimal" group of Tits in [29]. The rank of the Kac-Moody group of Sec. 6 is defined to be $l=|I|$ as in Sec. 1 .

We let $\lambda$ be a dominant integral linear functional on $\mathfrak{h}$, and let $V^{\lambda}$ be the corresponding irreducible highest weight module. Arguing as in [27] we can use the $\mathbb{Z}$-form $\mathcal{U}_{\mathbb{Z}}$ of $\mathcal{U}$ (as in $[29$, p. 78$]$ to obtain a $\mathbb{Z}$-form $V_{\mathbb{Z}}^{\lambda} \subseteq V^{\lambda}$ such that the following properties ((5.1) and (5.2)) hold:

$$
\begin{aligned}
& \frac{e_{i}^{m}}{m !} V_{\mathbb{Z}}^{\lambda} \subseteq V_{\mathbb{Z}}^{\lambda}, \\
& \frac{f_{i}^{m}}{m !} V_{\mathbb{Z}}^{\lambda} \subseteq V_{\mathbb{Z}}^{\lambda},
\end{aligned}
$$

for $m \in \mathbb{Z}_{\geq 0}$, and $i \in I$. We remark that for an irreducible highest weight module $V^{\lambda}$, the $e_{i}$ and $f_{i}$ are "locally nilpotent" on $V^{\lambda}$ (see [16, Chapt. 10]), that is for any $v \in V^{\lambda}, e_{i}^{n}(v)=0$ and $f_{i}^{n}(v)=0$ for almost all $n \in \mathbb{Z}_{\geq 0}$.

For each weight $\mu$ of $V^{\lambda}$, we let $V_{\mu}^{\lambda}$ be the corresponding weight space, and we set

$$
V_{\mu, \mathbb{Z}}^{\lambda}:=V_{\mu}^{\lambda} \cap V_{\mathbb{Z}}^{\lambda}
$$

We have

$$
V_{\mathbb{Z}}^{\lambda}=\oplus_{\mu} V_{\mu, \mathbb{Z}}^{\lambda}
$$

where the sum is taken over the weights of $V^{\lambda}$. We let $K$ denote a field, and we set

$$
\begin{aligned}
V_{K}^{\lambda} & :=K \otimes_{\mathbb{Z}} V_{\mathbb{Z}}^{\lambda}, \\
V_{\mu, K}^{\lambda} & :=K \otimes_{\mathbb{Z}} V_{\mu, K}^{\lambda},
\end{aligned}
$$

so that

$$
V_{K}^{\lambda}=\oplus_{\mu} V_{\mu, K}^{\lambda}
$$


For $u \in K$, we set

$$
\begin{aligned}
\chi_{\alpha_{i}}(u) & :=\sum_{m \in \mathbb{Z}_{\geq 0}} u^{m} \frac{e_{i}^{m}}{m !} \quad\left(=\exp \left(u e_{1}\right)\right), \\
\chi_{-\alpha_{i}}(u) & :=\sum_{m \in \mathbb{Z}_{\geq 0}} u^{m} \frac{f_{i}^{m}}{m !} \quad\left(=\exp \left(u f_{1}\right)\right) .
\end{aligned}
$$

Then $\chi_{\alpha_{i}}(u), \chi_{-\alpha_{i}}(u)$ define elements in $\operatorname{Aut}\left(V_{K}^{\lambda}\right)$ (thanks to the local nilpotence of $\left.e_{i}, f_{i}\right)$. We let $G_{0} \subseteq \operatorname{Aut}\left(V_{K}^{\lambda}\right)$ be the subgroup generated by the linear automorphisms $\chi_{ \pm \alpha_{i}}(u)$ of $V_{K}^{\lambda}$, for $i \in I, u \in K$. For $u \in K^{\times}, i \in I$, we set

$$
\begin{aligned}
w_{\alpha_{i}}(u) & :=\chi_{\alpha_{i}}(u) \chi_{-\alpha_{i}}\left(-u^{-1}\right) \chi_{\alpha_{i}}(u), \\
h_{\alpha_{i}}(u) & :=w_{\alpha_{i}}(u) w_{\alpha_{i}}(1)^{-1} .
\end{aligned}
$$

With a harmless abuse of notation, we set $w_{\alpha_{j}}:=w_{\alpha_{j}}(1)$ for each $j \in I$.

We let $H \subseteq G_{0}$ be the subgroup generated by the elements $h_{\alpha_{i}}(u), u \in K^{\times}$, $i \in I$. For Weyl roots $\alpha \in \Phi$ with $\alpha=w \alpha_{j}$, for some $w \in W, j \in I$, we define elements $\chi_{\alpha}(u), u \in K$ by

$$
\chi_{\alpha}(u):=\chi_{w \alpha_{j}}(u)=w \chi_{\alpha_{j}}(u) w^{-1} .
$$

( $\chi_{\alpha}(u)$ has an expression analogous to (5.3) where $e_{i}$ is replaced by its $w$-conjugate).

We note that $V_{\mathbb{Z}}^{\lambda}$ admits a coherently ordered basis which is defined as follows. For each weight $\mu$ of $V^{\lambda}, \mu=\lambda-\sum_{i=1}^{l} k_{i} \alpha_{i}$, where $k_{i} \in \mathbb{Z}_{\geq 0}$. Define the depth of $\mu$ to be

$$
\operatorname{depth}(\mu)=\sum_{i=1}^{l} k_{i}
$$

A basis $\Psi=\left\{v_{1}, v_{2}, \ldots\right\}$ of $V^{\lambda}$ is called coherently ordered if

(1) $\Psi$ consists of weight vectors.

(2) If $v_{i} \in V_{\mu}^{\lambda}, v_{j} \in V_{\mu^{\prime}}^{\lambda}$ and $\operatorname{depth}\left(\mu^{\prime}\right)>\operatorname{depth}(\mu)$, then $j>i$.

(3) $\Psi \cap V_{\mu}^{\lambda}$ consists of an interval $v_{k}, v_{k+1}, \ldots, v_{k+m}$.

Relative to a coherently ordered basis, the elements of $H$ are represented by diagonal matrices, the elements $\chi_{\alpha}(u), u \in K, \alpha \in \Phi^{+}$by upper triangular, unipotent matrices, and the elements $\chi_{\alpha}(u), u \in K, \alpha \in \Phi^{-}$by lower triangular, unipotent matrices.

\section{Kac-Moody Groups}

For each Weyl root $\alpha \in \Phi$, we define the root group $U_{\alpha}$ to be the group:

$$
U_{\alpha}:=\left\{\chi_{\alpha}(u) \mid u \in K\right\}, \quad \alpha \in \Phi,
$$


and we let $B_{\alpha}$ be the group generated by $U_{\alpha}$ and $H$. Then $U_{\alpha} \subseteq B_{\alpha}$ is a normal subgroup and $B_{\alpha}$ is the semi-direct product of $H$ and $U_{\alpha}$. One can then establish the following axioms (see [30, p. 562]):

$\left(\mathrm{RD}^{\prime}\right)$ Let $(\alpha, \beta)$ be a prenilpotent pair (see [30, p. 562] and (6.1) below). Let

$$
] \alpha, \beta\left[\operatorname{lin}=\left\{n \alpha+m \beta \in \Phi \mid n, m \in \mathbb{Z}_{>0}\right\} .\right.
$$

Then the commutator of $U_{\alpha}$ and $U_{\beta}$ is contained in the group generated by the $\left.U_{\gamma}, \gamma \in\right] \alpha, \beta[$ lin .

(RD2) For $i \in I$, and corresponding simple root $\alpha_{i}$, we have:

$$
B_{\alpha_{i}} \cap B_{-\alpha_{i}}=H .
$$

(RD3) The group $B_{\alpha_{i}}$ has two double cosets in the group $\left\langle B_{\alpha_{i}}, B_{-\alpha_{i}}\right\rangle$.

(RD4) There is an element in $\left\langle B_{\alpha_{i}}, B_{-\alpha_{i}}\right\rangle$ which, for each $\beta \in \Phi$, conjugates $B_{\beta}$ to $B_{w_{i} \beta}$, where $w_{i}$ is the Weyl reflection corresponding to $\alpha_{i}$.

(RD5) For each simple root $\alpha_{i}, B_{\alpha_{i}}$ is not contained in $B^{-}$, and $B_{-\alpha_{i}}$ is not contained in $B^{+}$, where $B^{ \pm}$is the group generated by the $B_{\beta}, \beta \in \Phi^{ \pm}$.

We remark that the verification of these axioms is straightforward, and we need only comment that we can obtain $\left(\mathrm{RD}^{\prime}\right)$ using the arguments of [27, Lemma 15] and its corollary (p. 24).

Recall from [30, p. 562] that $(\alpha, \beta) \in \Phi$ is a prenilpotent pair if there exist elements $w, w^{\prime} \in W$ such that

$$
\begin{array}{r}
w \cdot \alpha, \quad w \cdot \beta \in \Phi^{+}, \\
w^{\prime} \cdot \alpha, \quad w^{\prime} \cdot \beta \in \Phi^{-} .
\end{array}
$$

We let $S_{I} \subset W$ be the set of simple root reflections (so $w_{i} \in S_{I}, i \in I$ will be the reflection corresponding to the simple root $\alpha_{i}$ ). We let $U_{0}^{ \pm}$be the subgroup of $G_{0}$ generated by the $U_{\alpha}, \alpha \in \Phi^{ \pm}$. We let $B_{0}^{ \pm}$be the subgroup of $G_{0}$ generated by the $B_{\beta}, \beta \in \Phi^{ \pm}$, and we use the following notation:

$$
\begin{aligned}
U^{-} & :=U_{0}^{-}, \\
B^{-} & :=B_{0}^{-}, \\
B_{0} & :=B_{0}^{+}, \\
U_{0} & :=U_{0}^{+} .
\end{aligned}
$$

We have

$$
\begin{gathered}
B_{0}=H U_{0}, \\
B^{-}=H U^{-} .
\end{gathered}
$$

It follows that $B_{0} \cap B^{-}=H$ (just use a coherently ordered basis). 
We fix $m_{i} \in\left\langle B_{\alpha_{i}}, B_{-\alpha_{i}}\right\rangle$ so that

$$
m_{i} B_{\beta} m_{i}^{-1}=B_{w_{i} \cdot \beta}, \quad \beta \in \Phi,
$$

as in (RD4). We let $N$ be the subgroup of $G_{0}$ generated by $H$ and $\left\{m_{i}\right\}_{w_{i} \in S_{I}}$. From [30], we have

Proposition 6.1 ( $\left[30\right.$, Sec. 5]). $\left(G_{0}, B_{0}, N\right)$, and $\left(G_{0}, B^{-}, N\right)$ are $B N$-pairs, in fact twin $B N$-pairs in the sense of [31]. In particular, we have:

$$
\begin{aligned}
G_{0} & =B_{0} N B_{0} \\
& =B^{-} N B^{-} \\
& =B^{-} N B_{0} \\
& =B_{0} N B^{-} .
\end{aligned}
$$

Next we consider the completion of $G_{0}$, and we take $K=\mathbb{F}_{q}$, a finite field. In $V_{K}^{\lambda}$ we choose a coherently ordered basis $\Xi=\left\{v_{1}, v_{2}, \ldots\right\}$, so that

(i) $B_{0}$ is represented by upper triangular matrices with respect to $\Xi$.

(ii) $B^{-}$is represented by lower triangular matrices with respect to $\Xi$.

(iii) $H$ is represented by diagonal matrices with respect to $\Xi$.

We let $V_{t}$ be the span of the $v_{u}$, for $v_{u} \in \Xi$ with $u \leq t$. Then

$$
B_{0} V_{t} \subseteq V_{t}, \quad \text { for each } t .
$$

We let $B_{t}$ be the image of $B_{0}$ in $\operatorname{Aut}\left(V_{t}\right)$. We then have surjective homomorphisms

$$
B_{t^{\prime}} \stackrel{\pi_{t t^{\prime}}}{\longrightarrow} B_{t}, \quad t^{\prime} \geq t,
$$

and we let $B$ (the completion of $B_{0}$ ) be the projective limit of the projective family:

$$
\left\{B_{t}, \pi_{t t^{\prime}}\right\} .
$$

Then $B$ is profinite. On the other hand, $G_{0}$ itself can be topologized as follows: for a base of neighborhoods of the identity, we take sets

$$
V_{\left(u_{1}, \ldots, u_{p}\right)}, \quad u_{1}, \ldots, u_{p} \in V_{K}^{\lambda},
$$

defined by

$$
V_{\left(u_{1}, \ldots, u_{p}\right)}=\left\{g \in G_{0} \mid g u_{t}=u_{t}, t=1, \ldots, p\right\} .
$$

We take the corresponding completion $G$ of $G_{0}$, keeping groups $B^{-}, H, N$ as before. Then $G$ is a locally compact group, totally disconnected with $B$ an open profinite neighborhood of the identity, as follows from:

Lemma 6.1. Let $\left\{g_{t}\right\}_{t=1,2, \ldots}$ be a sequence in $G_{0}$ such that

$$
g_{t} \longrightarrow g \in G
$$


(in the above topology). Then there exists $g^{\prime} \in G_{0}$ and $t_{0} \in \mathbb{Z}_{\geq 0}$ so that for all $t \geq t_{0}$

$$
g_{t}=\left(g^{\prime}\right) \bmod B_{0}
$$

Proof. Let $v_{\lambda} \in V_{K}^{\lambda}$ be a highest weight vector $\left(v_{\lambda}=1 \otimes v_{\lambda}, v_{\lambda} \in V_{\mathbb{Z}}^{\lambda}\right)$. Then the sequence $g_{t} v_{\lambda}$ is eventually constant. Say $g_{t} v_{\lambda}=g_{t_{0}} v_{\lambda}, t \geq t_{0}$. For simplicity, we assume that $\lambda$ is regular, that is:

$$
\lambda\left(h_{i}\right)>0, \quad i=1, \ldots, l,
$$

( $h_{i}$ being the simple coroots). We have

$$
g_{t}^{-1} g_{t_{0}} v_{\lambda}=v_{\lambda}, \quad t \geq t_{0},
$$

and since $\lambda$ is regular,

$$
g_{t}^{-1} g_{t_{0}} \in B_{0}, \quad t \geq t_{0},
$$

as follows from the Bruhat decomposition $G_{0}=B_{0} W B_{0}$ obtained from [30, Proposition 1.1].

For $w_{i} \in S_{I}$, set

$$
U_{0, w_{i}}=m_{i} U_{0}^{+} m_{i}^{-1} \cap U_{0}^{+} .
$$

Lemma 6.2. If $u \in U_{0}^{+}$, and

$$
u \in m_{i} B_{0} m_{i}^{-1},
$$

then

$$
u \in m_{i} U_{0}^{+} m_{i}^{-1}
$$

Proof. Since

$$
B_{0}=H U_{0},
$$

and $m_{i}$ normalizes $H$, we have

$$
m_{i} B_{0} m_{i}^{-1}=H\left(m_{i} U_{0} m_{i}^{-1}\right) .
$$

Hence, by (6.3), $u$ has an expression

$$
u=h m_{i} u^{\prime} m_{i}^{-1}, \quad h \in H, u^{\prime} \in U_{0} .
$$

For each $q \in \mathbb{Z}_{>0}$, consider the set

$$
m_{i} v_{1}, \ldots, m_{i} v_{q}
$$

and the subspace $V(q) \subseteq V_{K}^{\lambda}$ spanned by the vectors (6.5).

The subspace $V(q)$ is $u$-invariant, and $u$ restricted to $V(q)$ is upper triangular with respect to the basis (6.5), thanks to the expression (6.4). Enlarge $V(q)$ to a finite dimensional subspace $\tilde{V}(q)$ by adding all weight spaces $V_{\mu, K}^{\lambda}$ with $\mu$ of smaller 
depth than the weight of some $m_{i} v_{j}, j=1, \ldots, q$. Then $\tilde{V}(q)$ is $u$-invariant, and $u$ acts on $\tilde{V}(q)$ as a unipotent linear transformation. Hence $u$ restricted to $V(q)$ is unipotent, and hence $\left.h\right|_{V(q)}$ is the identity. Since $q$ was arbitrary, $h$ is the identity on $V_{K}^{\lambda}$, and $u=m_{i} u^{\prime} m_{i}^{-1}$.

We now prove

Lemma 6.3. Assume that $\lambda$ is regular $\left(\lambda\left(\alpha_{i}^{\nu}\right)>0, i=1, \ldots, q\right)$. Then $U_{0}$ is the semi-direct product

$$
U_{0}=U_{\alpha_{i}} \ltimes U_{0, w_{i}} .
$$

Proof. We let $U^{\prime} \subseteq U_{0}$ be the normal subgroup generated by the $U_{\alpha}, \alpha \in \Phi^{ \pm}\left\{\alpha_{i}\right\}$, that is, $U^{\prime}$ is the smallest normal subgroup of $U_{0}$ containing all such $U_{\alpha}$. Then it is clear that

$$
U_{0}=U_{\alpha_{i}} U^{\prime}
$$

We consider an element

$$
u=\chi_{\alpha_{i}}(s) \chi_{\beta}\left(s^{\prime}\right) \chi_{\alpha_{i}}(-s), \quad s, s^{\prime} \in K,
$$

where $\beta$ is a positive Weyl root distinct from $\alpha_{i}$. For $v_{1}$, we take a highest weight vector $v_{\lambda}$ of $V_{K}^{\lambda}$. We have

$$
m_{i} v_{\lambda}=v_{w_{i} \lambda}
$$

a weight vector corresponding to the weight

$$
w_{i} \lambda=\lambda-k \alpha_{i}, \quad \text { some } k \in \mathbb{Z}_{>0},
$$

then

$$
\begin{aligned}
\chi_{\alpha_{i}}(-s) m_{i} v_{\lambda} & =\chi_{\alpha_{i}}(-s) v_{\lambda-k \alpha_{i}} \\
& \in V^{\prime}
\end{aligned}
$$

where

$$
V^{\prime}=\bigoplus_{j=0}^{k} V_{\lambda-j \alpha_{i}, K}^{\lambda} .
$$

We observe that $\chi_{\beta}\left(s^{\prime}\right)$ leaves each element of $V^{\prime}$ invariant. Hence

$$
\chi_{\alpha_{i}}(s) \chi_{\beta}\left(s^{\prime}\right) \chi_{\alpha_{i}}(-s) m_{i} v_{\lambda}=m_{i} v_{\lambda},
$$

and so

$$
m_{i}^{-1} \chi_{\alpha_{i}}(s) \chi_{\beta}\left(s^{\prime}\right) \chi_{\alpha_{i}}(-s) m_{i} \in B_{0}
$$

( $\lambda$ being regular, we apply the Bruhat decomposition). 
14 L. Carbone \&S H. Garland

By Lemma 6.2,

$$
m_{i}^{-1} \chi_{\alpha_{i}}(s) \chi_{\beta}\left(s^{\prime}\right) \chi_{\alpha_{i}}(-s) m_{i} \in U_{0},
$$

and so

$$
u \in U_{0} \cap m_{i} U_{0} m_{i}^{-1}=U_{0, w_{i}} .
$$

It follows that

$$
U^{\prime} \subseteq U_{0, w_{i}}
$$

and hence from (6.6), we have

$$
U_{0}=U_{\alpha_{i}} U_{0, w_{i}} .
$$

Now we have from the above argument

$$
u^{\prime} v_{w_{i} \lambda}=v_{w_{i} \lambda}, \quad u^{\prime} \in U^{\prime}
$$

(indeed we have verified this for a generating set of $U \prime$ ), while for $s \neq 0$

$$
\chi_{\alpha_{i}}(s) v_{w_{i} \lambda} \neq v_{w_{i} \lambda} .
$$

Hence

$$
U_{0}=U_{\alpha_{i}} \ltimes U^{\prime}
$$

If $u \in U_{0, w_{i}}$, then

$$
u=u_{1} u_{2}, \quad u_{1} \in U_{\alpha_{i}}, u_{2} \in U^{\prime},
$$

and by (6.7), $u_{2} \in U_{0, w_{i}}$. Hence $u_{1} \in U_{0, w_{i}}$, which is impossible, unless $u_{1}$ is the identity.

This shows that $U_{0, w_{i}}=U^{\prime}$, and hence by (6.9)

$$
U_{0}=U_{\alpha_{i}} \ltimes U_{0, w_{i}},
$$

which proves the lemma.

From now on, we will assume that $\lambda$ is regular.

Now let $U^{+}$be the closure of $U_{0}$ in $G$. If

$$
u_{t} \longrightarrow u \in U^{+},
$$

for $u_{t} \in U_{0}^{+}$, then eventually the sequences $u_{t} v_{\lambda}$ and $u_{t} m_{i} v_{\lambda}$ are constant, and by the above argument, for some $t_{0}$

$$
u_{t}=u_{t_{0}} \bmod U_{0, w_{i}}, \quad t \geq t_{0} .
$$

(First $u_{t}=u_{t_{0}} \bmod B_{0} \cap m_{i} B_{0} m_{i}^{-1}$, and then apply Lemma 6.2). It follows that if $U_{w_{i}}$ is the closure of $U_{0, w_{i}}$, then

$$
U^{+}=U_{\alpha_{i}} \ltimes U_{w_{i}},
$$

for each $w_{i} \in S_{I}$. 
One can then verify easily

Theorem 6.1. $(G, B, N)$ is a $B N$-pair, and also

$$
G=B N B^{-}=B^{-} N B .
$$

Proof. We show that

$$
w_{i} B w \subseteq B w_{i} w B \cup B w B .
$$

But since $w_{i} H w_{i}^{-1}=H$, it suffices to show that

$$
w_{i} U^{+} w \subseteq B w_{i} w B \cup B w B .
$$

But

$$
U^{+}=U_{\alpha_{i}} U^{\prime}, \quad U^{\prime}=U_{w_{i}}
$$

so if $u \in U^{+}$, then

$$
u=u^{\prime} u_{\alpha_{i}}, \quad u_{\alpha_{i}} \in U_{\alpha_{i}}, u^{\prime} \in U^{\prime},
$$

and

$$
\begin{aligned}
w_{i} u w & =w_{i} u^{\prime} u_{\alpha_{i}} w \\
& =\left(w_{i} u^{\prime} w_{i}^{-1}\right) w_{i} u_{\alpha_{i}} w, \quad w_{i} u w_{i}^{-1} \in B,
\end{aligned}
$$

(since $U^{\prime}=U_{w_{i}}$ ). But then we are reduced to showing

$$
w_{i} u_{\alpha_{i}} w \in B w_{i} w B \cup B w B,
$$

which follows from [30, Sec. 5] $\left(\left(G_{0}, B_{0}, N\right)\right.$ is a $B N$-pair $)$. The last two equalities of the theorem follow from the corresponding equalities for $G_{0}$ (see [30]) together with Lemma 6.1 above.

Remark 6.1. We have

$$
\begin{aligned}
B & =H U, & & \left(\text { where } U=U^{+}\right) \\
B^{-} & =H U^{-}, & & (\text {as we noted earlier })
\end{aligned}
$$

and hence $B \cap B^{-}=H$, as follows by considering a coherently ordered basis of $V_{K}^{\lambda}$.

The $B N$-pair structure on $G(6.13)$ gives an amalgam decomposition for $G$, where the factors are the standard parabolic subgroups.

Theorem 6.2 (Tits; [27, p. 92]). Let $(G, B, N)$ be a $B N$-pair. Let $S$ be the standard generating set for the Weyl group $W:=N /(B \cap N)$ consisting of simple root reflections. For each $s \in S$ let $W_{s}$ be the subgroup of $W$ generated by the element s. Let $G_{s}:=B W_{s} B=\bigcup_{w \in W_{s}} B w B$ be the corresponding standard parabolic subgroup. Then $G_{s}$ is a subgroup of $G$, and $G$ is the free product of $N$ and the $G_{s}$, $s \in S$, amalgamated along their intersections. 
16 L. Carbone \&3 H. Garland

\section{Existence of Lattices}

Let $G$ be a locally compact group with (left) Haar measure $\mu$. Let $\Gamma$ be a discrete subgroup. Then $\Gamma \backslash G$ carries a measure, also denoted $\mu$. We call $\Gamma$ a lattice if $\mu(\Gamma \backslash G)<\infty$, and a uniform lattice if $\Gamma \backslash G$ is compact, a non-uniform lattice otherwise.

To discuss the existence of lattices in $G$, we begin with the following general lemma.

Lemma 7.1. Let $G$ be a locally compact group with an open compact subgroup $B$ such that $G$ is the smallest normal subgroup containing $B$. Let $\Gamma \subseteq G$ be a discrete subgroup of $G$. We set $X=G / B$. Then $\Gamma$ has a natural left action on $X$. Let $S \subseteq X$ be a subset such that

$$
\Gamma S=X
$$

Then

(a) The isotropy group $\Gamma_{x}, x \in X$, is finite.

(b) If the series

$$
\sum_{x \in S} \frac{1}{\left|\Gamma_{x}\right|}
$$

is convergent, then $\Gamma \backslash G$ has finite invariant volume.

(The volume refered to here is the volume with respect to some (and hence any) Haar measure on $G$. Our assumptions on $B$ imply that $G$ is unimodular.)

Proof. Let

$$
\tilde{\omega}: G \longrightarrow G / B
$$

denote the natural projection, and let

$$
\tilde{S}=\tilde{\omega}^{-1}(S) .
$$

Then $S$ is an open subset of $G$, and

$$
\Gamma \tilde{S}=G,
$$

by (7.1). Let $\pi: G \longrightarrow \Gamma \backslash G$ denote the natural projection. Then

$$
\pi(\tilde{S})=\Gamma \backslash G,
$$

by $(7.2)$, and

$$
\pi(\tilde{S})=\bigcup_{x \in S} \pi\left(\tilde{S}_{x}\right)
$$

where $\tilde{S}_{x}=\tilde{\omega}^{-1}(x), x \in X=G / B$. In fact for $x \in X$, each set $\tilde{S}_{x}$ is a coset of the form $x^{\prime} B$, with $x^{\prime} \in G$ and $\tilde{\omega}\left(x^{\prime}\right)=x$. Moreover,

$$
\Gamma_{x}=\Gamma \cap x^{\prime} B\left(x^{\prime}\right)^{-1},
$$


which is a discrete subgroup of the compact group $x^{\prime} B\left(x^{\prime}\right)^{-1}$, and hence is finite. This proves (a). Now (7.3) and (7.4) imply that

$$
\text { volume }(\Gamma \backslash G) \leq \sum_{x \in S} \text { volume } \pi\left(\Gamma_{x} \backslash \tilde{S}_{x}\right)=\sum_{x \in S} \frac{1}{\left|\Gamma_{x}\right|},
$$

where "volume" is the volume with respect to a Haar measure on $G$, normalized so that the compact open subgroup $B$ has measure 1. Assertion (b) then follows.

\section{Non-Uniform "Arithmetic" Lattices}

We now let $G$ be a complete Kac-Moody group over a finite field, denoted $k$, and let $B, B^{-}, W$ be as in Sec. 6 . In this section, we show how to construct a non-uniform lattice in $G$. We have the decomposition from Theorem 6.1:

$$
G=B^{-} N B \text {. }
$$

To apply Lemma 7.1, we take $B^{-}=\Gamma, S=W B / B$, and using (8.1), we see that condition (7.1) is satisfied. We let $\Gamma_{w}, w \in W$, denote the isotropy group of the coset $w B$ :

$$
\Gamma_{w}=\{\gamma \in \Gamma \mid \gamma w B=w B\} .
$$

We wish to compute the orders $\left|\Gamma_{w}\right|$ of the groups $\Gamma_{w}, w \in W$, in particular showing that the groups $\Gamma_{w}$ are finite. We also wish to determine if

$$
\sum_{w \in W} \frac{1}{\left|\Gamma_{w}\right|}<\infty
$$

If the $\Gamma_{w}$ are finite, and (8.2) holds, then we can apply Lemma 7.1 to conclude that $\Gamma$ is a discrete subgroup of $G$ with finite covolume. In fact, we shall show:

Lemma 8.1. If $q=|k|$, then

$$
\left|\Gamma_{w}\right|=q^{l(w)}(q-1)^{l},
$$

where $l(\cdot)$ is the length function of the Weyl group $W$.

Assume Lemma 8.1. If $G$ is constructed using the $l \times l$ symmetrizable generalized Cartan matrix $A$, then $W$ is generated by simple root reflections $w_{1}, \ldots, w_{l}$, and the number of elements of length $r$ is $\leq l^{r}$. Hence if $q>l$, then

$$
\sum_{w \in W} \frac{1}{\left|\Gamma_{w}\right|}<\sum_{r=0}^{\infty} \frac{l^{r}}{q^{r}}
$$

which converges, provided $q>l$. Hence as a consequence of Lemmas 7.1 and 8.1, we have

Theorem 8.1. If $q>l$, then $\Gamma=B^{-}$is a discrete subgroup of $G$ with finite covolume. 
In case $l=2$, the number of elements in $W$ of length $r$ equals two (asuming that $W$ is infinite). In this case, we can drop the restriction on $q$, and we have

Theorem 8.2. If $l=2$, then $\Gamma=B^{-}$is a discrete subgroup of $G$ with finite covolume (with no restriction on $q$ ).

Proof of Lemma 8.1. First, for $w \in W$, we describe the cosets in

$$
B w B / B \text {. }
$$

We consider a reduced expression

$$
w^{-1}=w_{i_{1}} \cdots w_{i_{r}}, \quad(r=l(w))
$$

of $w^{-1}$, and the set of all roots $\alpha \in \Delta^{+}$such that

$$
w^{-1} \cdot \alpha \in \Delta^{-}
$$

that is, consider the set

$$
\Delta^{+} \cap w\left(\Delta^{-}\right) .
$$

Each simple root reflection $w_{i}$ corresponds to a simple root $\alpha_{i}$ of $\mathfrak{g}_{\mathbb{Q}}$. We then have (see [15, Proposition 2.2] and [22, Sec. 2]):

$$
\Delta^{+} \cap w\left(\Delta^{-}\right)=\left\{\alpha_{i_{r}}, w_{i_{r}} \cdot \alpha_{i_{r-1}}, \ldots, w_{i_{r}} \cdots w_{i_{m}} \cdot \alpha_{i_{m-1}}, \ldots, w_{i_{r}} \cdots w_{i_{2}} \cdot \alpha_{i_{1}}\right\} .
$$

Note that in particular,

$$
\left|\Delta^{+} \cap w\left(\Delta^{-}\right)\right|=r .
$$

The ordering of the elements in $\Delta^{+} \cap w\left(\Delta^{-}\right)$given in (8.6) will be singled out and called the "special ordering".

Lemma 8.2. For every $b^{\prime} \in B$, we have

$$
b^{\prime} w B=b w B,
$$

where $b$ has the form

$$
b=\Pi_{\alpha \in \Delta^{+} \cap w\left(\Delta^{-}\right)} \chi_{\alpha}\left(s_{\alpha}\right), \quad s_{\alpha} \in k
$$

(product taken with respect to the special ordering on $\Delta^{+} \cap w\left(\Delta^{-}\right)$). Moreover, if

$$
b^{\prime \prime}=\Pi_{\alpha \in \Delta^{+} \cap w\left(\Delta^{-}\right)} \chi_{\alpha}\left(s_{\alpha}^{\prime \prime}\right), \quad s_{\alpha}^{\prime \prime} \in k
$$

(product again taken with respect to the special ordering on $\Delta^{+} \cap w\left(\Delta^{-}\right)$), and if $b w B=b^{\prime \prime} w B$, then

$$
s_{\alpha}=s_{\alpha}^{\prime \prime}, \quad \text { for all } \alpha \in \Delta^{+} \cap w\left(\Delta^{-}\right) .
$$

Proof. We will argue by induction on the length of $w$. Assume that $w=w_{i}$ $(l(w)=1)$. If $b^{\prime} \in B$, then $b^{\prime}$ has an expression

$$
b^{\prime}=u h, \quad u \in U, h \in H
$$


and $u$ has an expression

$$
u=u_{1} u_{2},
$$

where $u_{1}=\chi_{\alpha_{i}}(s), s \in k, u_{2} \in U$ with $w_{i}^{-1} u_{2} w_{i} \in U$ (see Sec. 6). Then

$$
\begin{aligned}
b^{\prime} w B & =u_{1} u_{2} h w_{i} B \\
& =u_{1} w_{i} B
\end{aligned}
$$

(as $w_{i}^{-1} H w_{i}=H \subseteq B$ ). This proves the first assertion of the lemma for $l(w)=1$. On the other hand, if

$$
\chi_{\alpha_{i}}(s) w_{i} B=\chi_{\alpha_{i}}\left(s^{\prime}\right) w_{i} B, \quad s, s^{\prime} \in k,
$$

and if $s \neq s^{\prime}$, then we have

$$
\chi_{\alpha_{i}}(\tilde{s}) w_{i} B=w_{i} B
$$

for some $\tilde{s} \neq 0$. Take $\lambda$ regular (as in Lemma 6.3). If $V_{k}^{\lambda}$ is a highest weight module with highest weight $\lambda$, and $v_{\lambda} \neq 0$ is a highest weight vector, then

$$
w_{i} \tilde{b} \cdot v_{\lambda}=\tilde{v}_{w_{i} \lambda}, \quad \tilde{b} \in B
$$

where $\tilde{v}_{w_{i} \lambda}$ is a (non-zero) weight vector corresponding to the weight $w_{i} \lambda$. On the other hand,

$\chi_{\alpha_{i}}(\tilde{s}) w_{i} \cdot v_{\lambda}=v_{w_{i} \lambda}+$ weight vectors corresponding to weights higher than $w_{i} \lambda$

$\left(v_{w_{i} \lambda}=w_{i} \cdot v_{\lambda}\right.$, a non-zero weight vector corresponding to the weight $\left.w_{i} \lambda\right)$. The only way this can coincide with (8.5) is if there are no higher weight components. But then $\tilde{s}=0$, a contradiction.

Now we assume that we have proven the lemma for Weyl group elements of length $\leq r-1$, and assume that $l(w)=r$. Assume that we have a coset

$$
b^{\prime} w B,
$$

with $w^{-1}=w_{i_{1}} \cdots w_{i_{r}}, w=w_{i_{r}} \cdots w_{i_{1}}, b^{\prime} \in B$. Then

$$
b^{\prime} w B=b^{\prime} w_{i_{r}} w^{\prime} B,
$$

where $w^{\prime}=w_{i_{r-1}} \cdots w_{i_{1}}$. By the above case (Weyl group elements of length one), we have

$$
b^{\prime} w_{i_{r}}=\chi_{\alpha_{i_{r}}}(s) w_{i_{r}} b^{\prime \prime},
$$

for some $s \in k, b^{\prime \prime} \in B$. Therefore,

$$
b^{\prime} w_{i_{r}} w^{\prime} B=\chi_{\alpha_{i_{r}}}(s) w_{i_{r}} b^{\prime \prime} w^{\prime} B,
$$


20 L. Carbone \&S H. Garland

and by our inductive hypothesis,

$$
b^{\prime \prime} w^{\prime} B=\Pi_{\alpha \in \Delta^{+} \cap w^{\prime}\left(\Delta^{-}\right)} \chi_{\alpha}\left(s_{\alpha}^{\prime}\right) w^{\prime} B
$$

for some $s_{\alpha}^{\prime} \in k$ (the product taken with respect to the special ordering). Hence, (continuing with (8.6)), we obtain

$$
\begin{aligned}
b^{\prime} w_{i_{r}} w^{\prime} B & =\chi_{\alpha_{i_{r}}}(s) w_{i_{r}} \Pi_{\alpha \in \Delta^{+} \cap w^{\prime}\left(\Delta^{-}\right)} \chi_{\alpha}\left(s_{\alpha}^{\prime}\right) w^{\prime} B \\
& =\left[\chi_{\alpha_{i_{r}}}(s) \Pi_{\alpha \in \Delta^{+} \cap w^{\prime}\left(\Delta^{-}\right)} \chi_{w_{i_{r}} \cdot \alpha}\left( \pm s_{\alpha}^{\prime}\right)\right] w^{\prime} B
\end{aligned}
$$

and the expression in brackets is exactly of the desired form, so the induction is complete. Finally we must prove the uniqueness assertion of the lemma. Let

$$
\begin{aligned}
& u_{1}=\Pi_{\alpha \in \Delta^{+} \cap w\left(\Delta^{-}\right)} \chi_{\alpha}\left(s_{\alpha}\right) \\
& u_{2}=\Pi_{\alpha \in \Delta^{+} \cap w\left(\Delta^{-}\right)} \chi_{\alpha}\left(s_{\alpha}^{\prime \prime}\right)
\end{aligned}
$$

(products with respect to the special order on $\Delta^{+} \cap w\left(\Delta^{-}\right)$), and assume

$$
u_{1} w=u_{2} w b
$$

for some $b \in B$. Then

$$
w^{-1} u_{i} w \in U^{-}
$$

for $i=1,2$, and so,

$$
w^{-1} u_{2}^{-1} u_{1} w \in U^{-}
$$

From (8.8), we obtain

$$
w^{-1} u_{2}^{-1} u_{1} w=b,
$$

where, acting on a highest weight module $V_{k}^{\lambda}$ ( $\lambda$ dominant integral), $b$ is upper triangular, and $w^{-1} u_{2}^{-1} u_{1} w$ is lower triangular with ones on the diagonal (with respect to a coherently ordered basis) of $V_{k}^{\lambda}$ ). Hence both sides of (8.10) act as the identity on $V_{k}^{\lambda}$, and so as automorphisms on $V_{k}^{\lambda}$,

$$
u_{2}=u_{1} .
$$

Now we consider

$$
w_{i_{r}}^{-1} u_{1} w_{i_{r}} \cdot v_{\lambda}=w_{i_{r}}^{-1} \chi_{\alpha_{i_{r}}}\left(s_{\alpha_{i_{r}}}\right) w_{i_{r}} \cdot v_{\lambda}=v_{\lambda}+s_{\alpha_{i_{r}}} \cdot v_{\lambda-\alpha_{i_{r}}}+\cdots
$$

where $v_{\lambda-\alpha_{i_{r}}} \neq 0$ is a vector in the weight space $V_{\lambda-\alpha_{i_{r}}}^{\lambda}$ and similarly

$$
w_{i_{r}}^{-1} u_{2} w_{i_{r}} \cdot v_{\lambda}=v_{\lambda}+s_{\alpha_{i_{r}}}^{\prime \prime} \cdot v_{\lambda-\alpha_{i_{r}}}+\cdots,
$$

and hence by (8.11)

$$
s_{\alpha_{i_{r}}}=s_{\alpha_{i_{r}}}^{\prime \prime} .
$$


To see that $v_{\lambda-\alpha_{i_{r}}} \neq 0$, we consider $w_{i_{r}} \cdot v_{\lambda}$, which equals

$$
\pm\left(\frac{f_{i_{r}}^{n}}{n !}\right) \cdot v_{\lambda}, \quad n=\lambda\left(\alpha_{i_{r}}^{\nu}\right)
$$

thanks to [14], and the argument in the proof of [14, Lemma 2.9] therein. It follows that

$$
f_{i_{r}} \cdot v_{\lambda}=v_{\lambda-\alpha_{i_{r}}} \neq 0
$$

as well.

Hence from the expression (8.7), we have

$$
\begin{aligned}
& w_{i_{r}}^{-1} \chi_{\alpha_{i_{r}}}\left(s_{\alpha_{i_{r}}}\right)^{-1} u_{1} w_{i_{r}}^{-1}=u_{1}^{\prime}, \\
& w_{i_{r}}^{-1} \chi_{\alpha_{i_{r}}}\left(s_{\alpha_{i_{r}}}\right)^{-1} u_{2} w_{i_{r}}^{-1}=u_{2}^{\prime},
\end{aligned}
$$

where

$$
\begin{aligned}
& u_{1}^{\prime}=\Pi_{\alpha \in \Delta^{+} \cap w^{\prime}\left(\Delta^{-}\right)} \chi_{\alpha}\left( \pm s_{\alpha}\right), \\
& u_{2}^{\prime}=\Pi_{\alpha \in \Delta^{+} \cap w^{\prime}\left(\Delta^{-}\right)} \chi_{\alpha}\left( \pm s_{\alpha}^{\prime \prime}\right),
\end{aligned}
$$

(where the \pm signs in the two expressions match up " $\alpha$ by $\alpha$ "). By induction, $s_{\alpha}=$ $s_{\alpha}^{\prime \prime}, \alpha \in \Delta^{+} \cap w^{\prime}\left(\Delta^{-}\right)$, and since $s_{\alpha_{i_{r}}}=s_{\alpha_{i_{r}}}^{\prime \prime}$, we obtain $s_{\alpha}=s_{\alpha}^{\prime \prime}, \alpha \in \Delta^{+} \cap w\left(\Delta^{-}\right)$, which completes the induction, and hence the proof of uniqueness, and the proof of Lemma 8.2.

We are now ready to consider the equality

$$
\left|\Gamma_{w}\right|=q^{l(w)}(q-1)^{l},
$$

of Lemma 8.1. If $b^{-} \in B^{-}$, and

$$
B w b^{-}=B w,
$$

then for some $b \in B$,

$$
w b^{-} w^{-1}=b .
$$

Equivalently,

$$
w^{-1} b w=b^{-} \in B^{-} .
$$

Now by Lemma 8.2,

$$
b w B=u w B,
$$

for

$$
u=\Pi_{\alpha \in \Delta^{+} \cap w\left(\Delta^{-}\right)} \chi_{\alpha}\left(s_{\alpha}\right),
$$


$s_{\alpha} \in k$, (product taken with respect to the special ordering), and the $s_{\alpha}$ are uniquely determined. Hence

$$
b^{-}=w^{-1} b w=w^{-1} u w b^{\prime},
$$

for some $b^{\prime} \in B$. Now $b^{-}$has an expression

$$
b^{-}=u^{-} h,
$$

$u^{-} \in U^{-}, h \in H$, and so (8.13) becomes

$$
u^{-} h=w^{-1} u w b^{\prime} .
$$

Then

$$
\left(u^{-}\right)^{-1} w^{-1} u w=h\left(b^{\prime}\right)^{-1} \in B \cap U^{-} .
$$

By Remark 6.1, we have

$$
B \cap B^{-}=H,
$$

so

$$
\left(u^{-}\right)^{-1}\left(w^{-1} u w\right) \in H
$$

and hence

$$
u^{-}=w^{-1} u w .
$$

There are then exactly $q^{l(w)}$ choices for $u^{-}$, and then $(q-1)^{l}$ choices for $h$. This concludes the proof of Lemma 8.1, and hence of Theorems 8.1 and 8.2.

Let $P^{-} \subseteq G$ be a proper standard parabolic subgroup for the $B N$-pair $\left(G, B^{-}, N\right)$ (thus $\left.B^{-} \subseteq P^{-} \subsetneq G\right)$. Assume that the submatrix of $A$ corresponding to $P^{-}$is positive-definite. Then $B^{-} \backslash P^{-}$is finite. Hence, if $B^{-}$is a lattice, then so is $P^{-}$. Thus we obtain

Corollary $8.1([7,(\mathbf{1 . 7})])$. If $P^{-}$corresponds to a positive-definite submatrix of $A$ and $q>l$, then $P^{-}$is a non-uniform lattice in $G$.

\section{Bruhat-Tits Tree in Rank 2}

We now take $l=2$ and we assume that the Cartan matrix $A$ is symmetric and given by

$$
A=\left(\begin{array}{cc}
2 & -m \\
-m & 2
\end{array}\right)
$$

for $m \geq 2$. We let $(G, B, N)$ be the $B N$-pair as in Theorem 6.1. We have fixed subgroups $B \subseteq G, N \subseteq G$, and the Weyl group $W=N / H$, where $H=N \cap B$ is a normal subgroup of $N$. We let $S=\left\{w_{1}, w_{2}\right\}$ be the generators of $W$ corresponding to simple roots $\alpha_{1}$ and $\alpha_{2}$ respectively. Then $W$ is the infinite dihedral group

$$
W=\mathbb{Z} / 2 \mathbb{Z} * \mathbb{Z} / 2 \mathbb{Z} \cong \mathbb{Z} \rtimes\{ \pm 1\} .
$$


If we consider the Bruhat cells $B w_{1} B$ and $B w_{2} B$ from (6.13), we obtain subgroups

$$
\begin{aligned}
& P_{1}:=B \sqcup B w_{1} B, \\
& P_{2}:=B \sqcup B w_{2} B
\end{aligned}
$$

of $G$, which will be called standard parabolic subgroups. The subgroup $B \subseteq G$ will be called a minimal parabolic subgroup.

The Bruhat-Tits building of $(G, B, N)$ is a simplicial complex $X$ of dimension

$$
\operatorname{dim}(X)=|S|-1=1 .
$$

Since $W$ is infinite, by the Solomon-Tits theorem (see [12, Theorem 2.2]) $X$ is contractible, and so $X$ is a tree. The vertices of $X$ are the conjugates of $P_{1}$ and $P_{2}$ in $G$. If $Q_{1}$ and $Q_{2}$ are vertices, then there is an edge connecting $Q_{1}$ and $Q_{2}$ if and only if $Q_{1} \cap Q_{2}$ contains a conjugate of $B$. We have an action of $G$ on $X$ by conjugation. The vertex set of $X$ will be denoted $V X$ and the set of oriented edges by $E X$. We will make use of the following basic facts:

(1) $P_{1}$ and $P_{2}$ are not conjugate in $G$.

(2) Each $P_{i}$ is its own normalizer in $G, i=1,2$, and $B$ is its own normalizer in $G$.

Let $g \in G$. Then (1) and (2) imply that the maps:

$$
\begin{aligned}
& f_{0}: g B \longrightarrow g B g^{-1}, \\
& f_{1}: g P_{1} \longrightarrow g P_{1} g^{-1}, \\
& f_{2}: g P_{2} \longrightarrow g P_{2} g^{-1}
\end{aligned}
$$

are bijections. Thus

$$
\begin{aligned}
& G / P_{1} \cong \text { all conjugates of } P_{1} \text { in } G, \\
& G / P_{2} \cong \text { all conjugates of } P_{2} \text { in } G, \\
& G / B \cong \text { all conjugates of } B \text { in } G .
\end{aligned}
$$

Conjugates of $P_{1}, P_{2}$ will be called parabolic subgroups. (In the affine case, these are often called parahoric subgroups.) Applying the fundamental theory of Bass-Serre [28], we obtain

$$
G=P_{1} *_{B} P_{2} .
$$

We obtain the following description of the Bruhat-Tits tree $X$. We have bijective correspondences

$$
\begin{aligned}
& V X \cong G / P_{1} \sqcup G / P_{2} \\
& E X \cong G / B \sqcup \overline{G / B},
\end{aligned}
$$


where $\overline{G / B}$ is a copy of the set $G / B$, giving an orientation to EX so that positively oriented edges come from $G / B$, and negatively oriented edges come from $\overline{G / B}$.

There is a standard simplex $\mathcal{C}_{0}$ corresponding to the identity coset of $B$. Let $P$ be a parabolic subgroup. The corresponding simplex (vertex or edge) will be denoted $\sigma_{P}$. We may at times identify a simplex with it's corresponding parabolic subgroup.

The group $G$ acts by left multiplication on cosets. There are natural projections on cosets induced by the inclusion of $B$ in $P_{1}$ and $P_{2}$ :

$$
\pi: G / B \longrightarrow G / P_{i}, \quad i=1,2 .
$$

If $v_{i} \in G / P_{i}$ is a vertex, and $S t^{X}\left(v_{i}\right)=\pi^{-1}\left(v_{i}\right)$ is the set of edges with origin $v_{i}$, then we may index $S t^{X}\left(v_{i}\right)$ by $P_{i} / B \subseteq G / B, i=1,2$ (however, the choice of indexing is not unique). The Bruhat-Tits tree $X$ is a homogeneous, bipartite tree of degree

$$
\left[P_{1}: B\right]=\left[P_{2}: B\right]=|k|+1 .
$$

Adapting the discussion in the previous section to the case $l=2$, the following describes how the cosets $B w_{1} B$ and $B w_{2} B$ are indexed modulo $B$ :

\section{Lemma 9.1.}

$$
\begin{aligned}
& B w_{1} B / B=\left\{\chi_{\alpha_{1}}(s) w_{1} B / B \mid s \in k\right\}, \\
& B w_{2} B / B=\left\{\chi_{\alpha_{2}}(s) w_{2} B / B \mid s \in k\right\},
\end{aligned}
$$

where $\alpha_{1}$ and $\alpha_{2}$ are the simple roots corresponding to $w_{1}$ and $w_{2}$ respectively.

It follows that the edges emanating from $P_{1}$ and $P_{2}$ may be indexed as follows:

$$
\begin{aligned}
& S t^{X}\left(P_{1}\right)=\{B\} \cup\left\{\chi_{\alpha_{1}}(s) w_{1} B / B \mid s \in k\right\}, \\
& S t^{X}\left(P_{2}\right)=\{B\} \cup\left\{\chi_{\alpha_{2}}(s) w_{2} B / B \mid s \in k\right\},
\end{aligned}
$$

where $B$ denotes the identity coset, and the stars of other vertices are obtained by translating (conjugating) these.

Apartments in $X$ are infinite lines. The standard apartment, denoted by $\mathcal{A}_{0}$, in $X$ consists of all Weyl group translates of the standard simplex. That is

$$
\begin{aligned}
& V \mathcal{A}_{0}=W \cdot \sigma_{P_{1}} \cup W \cdot \sigma_{P_{2}} \\
& E \mathcal{A}_{0}=W \cdot \sigma_{B} .
\end{aligned}
$$

It is worthwhile to picture $\mathcal{A}_{0}$. In the diagram below, it is to be understood that all products

$$
w^{\prime} w_{i}, \quad w^{\prime} \in W, \quad i=1,2
$$

have the property that

$$
l\left(w^{\prime} w_{i}\right)=l\left(w^{\prime}\right)+1 .
$$


We then have the following diagram of the apartment $\mathcal{A}_{0}$ :


Fig. 9.1. The tree of a field with 2 elements.

Since the subgroups $P_{1}$ and $P_{2}$ are not conjugate in $G$, the group $G$ acts without inversions on $X$. Also, $G$ acts transitively on edges of $X$, and has 2 orbits for the vertices, corresponding to vertices that come from $P_{1}$ and from $P_{2}$. Figure 9.1 shows the tree of the field with 2 elements. 
The group

$$
G /\left(\bigcap_{g \in G} g B g^{-1}\right)
$$

acts faithfully on $X$, and the defining homomorphism

$$
\rho: G /\left(\bigcap_{g \in G} g B g^{-1}\right) \hookrightarrow \operatorname{Aut}(X)
$$

is continuous and the image is closed. The quotient topology on $G /\left(\bigcap_{g \in G} g B g^{-1}\right)$ coincides with that induced by the natural topology on $\operatorname{Aut}(X)$, where the stabilizers of finite sets of vertices form a fundamental system of compact open neighborhoods of the identity. The group $B$ of the Tits system is the stabilizer of the standard simplex $\mathcal{C}_{0}$, and $N$ is contained in the stabilizer of the standard apartment $\mathcal{A}_{0}$.

\section{Schottky Groups of Tree Automorphisms}

In [18], Lubotzky adapted the classical notion of a Schottky group of automorphisms of the upper half plane to the non-archimedean case. We describe Lubotzky's Schottky groups of tree automorphisms in this section.

Let $X$ be any locally finite tree. Then $\operatorname{Aut}(X)$ contains three possible types of automorphisms. Let $g \in \operatorname{Aut}(X)$. Then [32] $g$ is either:

(1) an inversion, ( $g^{2}$ fixes a vertex, but $g$ fixes none),

(2) elliptic ( $g$ fixes a vertex),

(3) hyperbolic (both $g$ and $g^{2}$ fix no vertex).

Lubotzky constructed uniform lattices out of hyperbolic tree automorphisms. We have the following:

Proposition 10.1 ([32]). Let $X$ be a tree and let $g \in \operatorname{Aut}(X)$ be hyperbolic. Let $m=\min _{x \in V X} d(x, g x)$. Let $L=\{x \in V X \mid d(x, g x)=m\}$. Then $L$ is the vertex set of a bi-infinite path in $X$, and $g$ acts as a translation of length (amplitude) $m$ along $L$ ( $L$ is the "axis" of $g$ ).

Lubotzky has given the following criterion for recognizing hyperbolic elements:

Lemma 10.1 ([18, Lemma 1.2]). Let $x, y \in V X$ be such that $d(x, y)=1$ and suppose that there exists $\gamma \in \operatorname{Aut}(X)$ with

$$
d(x, \gamma x)=d(y, \gamma y)>0 .
$$

Then $\gamma$ is hyperbolic and $x$ and $y$ lie on the axis of $\gamma$.

Let $\gamma$ be hyperbolic of amplitude $m=m(\gamma)$ and with axis $L=L(\gamma)$. We may choose a labelling of the vertices of $L(\gamma)$ as $\left\{x_{n}, n \in \mathbb{Z}\right\}$ in such a way that 
$d\left(x_{n}, x_{n+1}\right)=1$, and $\gamma\left(x_{n}\right)=x_{n+m}$. We choose such a labelling and set

$$
\begin{aligned}
& A(\gamma)=\left\{x \in V X \mid d\left(x, x_{0}\right)<d\left(x, x_{1}\right)\right\}, \\
& B(\gamma)=\left\{x \in V X \mid d\left(x, x_{m+1}\right)<d\left(x, x_{m}\right)\right\} .
\end{aligned}
$$

Let $\gamma_{1}, \ldots, \gamma_{s}$ be hyperbolic elements in $\operatorname{Aut}(X)$. Assume that the axes $L\left(\gamma_{1}\right), \ldots, L\left(\gamma_{s}\right)$ can be labelled in such a way that the sets $A\left(\gamma_{i}\right), B\left(\gamma_{i}\right), i=$ $1, \ldots, s$ are mutually disjoint. Let $\Gamma$ be the group generated by $\left\{\gamma_{1}, \ldots, \gamma_{s}\right\}$. Then $\left\{\gamma_{1}, \ldots, \gamma_{s}\right\}$ is called a Schottky basis for $\Gamma$. We also call $\Gamma$ a Schottky group. We have the following:

Theorem 10.1 ([18, Proposition 1.6]). Let $\Gamma$ be generated by a Schottky basis $\gamma_{1}, \ldots, \gamma_{s}$. Then

(1) $\Gamma$ is discrete and every element $\neq 1$ of $\Gamma$ is hyperbolic.

(2) $\Gamma$ is a free group and $\gamma_{1}, \ldots, \gamma_{s}$ are free generators of $\Gamma$.

(3) $F=X-\left(\bigcup_{i=1}^{s}\left(A\left(\gamma_{i}\right) \cup B\left(\gamma_{i}\right)\right)\right.$ is a fundamental domain for $\Gamma$ on $X$.

Remark 10.1. (1) (1) in the theorem implies that $\Gamma$ acts freely on $X$ since no element $\neq 1$ of $\Gamma$ fixes a vertex.

(2) A Schottky group $\Gamma$ may be non-uniform, but if $\Gamma$ is a lattice, it is necessarily uniform.

Let $F=\left\langle s_{1}, \ldots, s_{r}\right\rangle$ be free of rank $r$. Let $X$ be a locally finite tree. Let $\rho$ : $F \longrightarrow \operatorname{Aut}(X)$ be a representation. We say that $\rho(F)$ admits a Schottky basis in $\operatorname{Aut}(X)$ if $\rho$ is faithful, and $\rho\left(s_{i}\right)=\gamma_{i}$, where $\left\langle\gamma_{1}, \ldots, \gamma_{r}\right\rangle$ is a Schottky group. We also call $\rho$ a Schottky representation.

Theorem 10.2 ([18, Proposition 1.7]). Let $X$ be a locally finite tree. Let $\Gamma$ be any torsion free discrete subgroup of $\operatorname{Aut}(X)$. Then $\Gamma$ admits a Schottky basis in $\operatorname{Aut}(X)$.

Proof. Since $\Gamma$ is torsion free, $\Gamma$ contains no elements of finite order, so $\Gamma$ must act without inversions. Let $p: X \longrightarrow \Gamma \backslash X$ be the quotient morphism. Then $\Gamma$ is discrete if and only if each vertex stabilizer $\Gamma_{x}$ is finite, for $x \in V X$. Hence $\Gamma$ discrete and torsion free implies that

$$
\Gamma_{x}=\{1\}, \quad \text { for all } x \in V X .
$$

Thus $\Gamma$ acts freely on $X$, and so $\Gamma \cong \pi_{1}(\Gamma \backslash X)$, a free group, say $\Gamma$ is free of rank $r \leq \infty$. To find a Schottky basis for $\Gamma$ in $\operatorname{Aut}(X)$, we form an opening (as in [28, Proposition 14]) of $\Gamma \backslash X$ in $X$ as follows: since the quotient morphism $p: X \longrightarrow \Gamma \backslash X$ is locally surjective (surjective on stars of vertices), there exist connected subgraphs $T \subseteq S \subseteq X$ such that:

(i) $T$ is a tree,

(ii) $p_{T}: T \longrightarrow \Gamma \backslash X$ is bijective on vertices and $p_{S}: S \longrightarrow \Gamma \backslash X$ is bijective on edges. 
(iii) For each $e \in E S$, either $\partial_{0} e$ or $\partial_{1} e$ belongs to $T$.

(iv) $|V S-V T|=r=\operatorname{rank}(\Gamma)$.

Denote the inverses of the bijections $p_{T}: V T \longrightarrow V(\Gamma \backslash X)$ and $p_{S}: E S \longrightarrow$ $E(\Gamma \backslash X)$ by

$$
\begin{aligned}
a & \mapsto a^{T}, \\
e & \mapsto e^{S},
\end{aligned}
$$

respectively.

For each $x \in V S$, choose $g_{x} \in \Gamma$ so that $g_{x} x \in V T$; that is, $g_{x} x=p(x)^{T}$, and so that $g_{x}=1$, if $x \in V T$. Then $\Gamma$ is free (of rank $r$ ) on

$$
\left\{g_{x} \mid x \in V S-V T\right\} .
$$

We claim that $\Gamma$ is a Schottky group. For each $x \in V S-V T, g_{x} \in \Gamma$ which acts freely on $X$, hence without fixed points, so each $g_{x}$ is hyperbolic. Let $m_{x}=m\left(g_{x}\right)$ be the amplitude of $g_{x}$ and let $L_{x}=L\left(g_{x}\right)$ be the axis of $g_{x}$. Choose a labelling of the vertices of $L_{x}$ as in (10.1), and define sets $A\left(g_{x}\right)$ and $B\left(g_{x}\right)$, as in (10.1). Since $T$ is a fundamental domain for vertices and $S$ is a fundamental domain for edges, it is a routine check that the subsets $A\left(g_{x}\right)$ and $B\left(g_{x}\right)$ are all disjoint, for $x \in V S-V T$.

Let $\Gamma\left\langle s_{1}, \ldots, s_{r}\right\rangle$ be free of rank $r$. Let $X$ be a locally finite tree, and let $\operatorname{Aut}(X)$. Theorem 10.2 gives rise to the following discussion of representation spaces of $\Gamma$ in $G$. Since $\Gamma$ is free,

$$
\operatorname{Hom}(\Gamma, \operatorname{Aut}(X)) \cong \operatorname{Aut}(X) \times \cdots \times \operatorname{Aut}(X)
$$

( $r$ factors). Let $\rho_{0}: \Gamma \longrightarrow \operatorname{Aut}(X)$ be a faithful discrete representation. Hence $\rho_{0}(\Gamma)$ acts freely on $X$. Choose $T \subseteq S \subseteq X$ such that $p_{T}: T \longrightarrow \Gamma \backslash X$ is bijective on vertices and $p_{S}: S \longrightarrow \Gamma \backslash X$ is bijective on edges. For each $x \in V S-V T$, choose $g_{x} \in \rho_{0}(\Gamma)$ such that $g_{x} x=p(x)^{T}$. Then $|V S-V T|=r$, and $\left\{g_{x_{1}}, \ldots, g_{x_{r}}\right\}$ generates $\rho_{0}(\Gamma)$ freely, and is a Schottky basis for $\rho_{0}(\Gamma)$. Let $L_{i}=L\left(g_{i}^{-1}\right)$ be the axis of $g_{i}^{-1}$. Let $f_{i} \in L_{i}$ and let $g_{i}^{-1}\left(f_{i}\right)=e_{i}$. Let

$$
\begin{array}{r}
\operatorname{Hom}_{0}(\Gamma, \operatorname{Aut}(X))=\{\phi \in \operatorname{Hom}(\Gamma, \operatorname{Aut}(X)) \mid \phi \text { is faithful } \\
\\
\left.\quad \text { and } \phi\left(s_{i}\right)\left(e_{i}\right)=f_{i}, i=1, \ldots, r\right\},
\end{array}
$$

and let

$$
\begin{aligned}
& \operatorname{Hom}_{1}(\Gamma, \operatorname{Aut}(X))=\{\phi \in \operatorname{Hom}(\Gamma, \operatorname{Aut}(X)) \mid \phi \text { is faithful } \\
&\text { and } \left.\phi\left(s_{i}\right) \in B_{i} g_{i}, i=1, \ldots, r\right\},
\end{aligned}
$$

where $B_{i}=\operatorname{Stab}_{\operatorname{Aut}(X)}\left(f_{i}\right)$.

Proposition 10.2. $\operatorname{Hom}_{1}(\Gamma, \operatorname{Aut}(X))=\operatorname{Hom}_{0}(\Gamma, \operatorname{Aut}(X))$ 
Proof. Let $\psi \in \operatorname{Hom}_{1}(\Gamma, \operatorname{Aut}(X))$. Thus

$$
\begin{aligned}
\psi\left(s_{i}\right)\left(e_{i}\right) & =b_{i} g_{i}\left(e_{i}\right), b_{i} \in B_{i} \\
& =b_{i} f_{i} \\
& =f_{i},
\end{aligned}
$$

and so $\psi \in \operatorname{Hom}_{0}(\Gamma, \operatorname{Aut}(X))$.

Conversely, let $\psi \in \operatorname{Hom}_{0}(\Gamma, \operatorname{Aut}(X))$. Then $\psi\left(s_{i}\right)^{-1}\left(f_{i}\right)=e_{i}$, but $g_{i}^{-1}\left(f_{i}\right)=e_{i}$, so

$$
g_{i} \psi\left(s_{i}\right)^{-1}\left(f_{i}\right)=f_{i},
$$

and so $g_{i} \psi\left(s_{i}\right)^{-1} \in B_{i}=\operatorname{Stab}_{\operatorname{Aut}(X)}\left(f_{i}\right)$. Therefore

$$
\psi\left(s_{i}\right)=b_{i} g_{i}, \text { some } b_{i} \in B_{i},
$$

and so $\psi \in \operatorname{Hom}_{1}(\Gamma, \operatorname{Aut}(X))$.

Proposition 10.3. $\operatorname{Hom}_{0}(\Gamma, \operatorname{Aut}(X))$ is open in $\operatorname{Hom}(\Gamma, \operatorname{Aut}(X))$. In particular, $\operatorname{Hom}_{0}(\Gamma, \operatorname{Aut}(X))$ contains all representations in an open neighbourhood of the inclusion map $\rho_{0}: \Gamma \longrightarrow \operatorname{Aut}(X)$.

Proof. Let $\psi: \Gamma \longrightarrow \operatorname{Aut}(X)$ be a faithful representation in an open neighbourhood of the inclusion map $\rho_{0}: \Gamma \longrightarrow \operatorname{Aut}(X)$. Using the topology on $\operatorname{Aut}(X)[3$, Chapt. 3], we see that $\psi$ and $\rho_{0}$ agree on the axis of $\rho_{0}\left(s_{i}\right), i=1, \ldots, r$. Thus $\psi \in \operatorname{Hom}_{0}(\Gamma, \operatorname{Aut}(X))$.

Thus $\operatorname{Hom}_{0}(\Gamma, \operatorname{Aut}(X))=\operatorname{Hom}_{1}(\Gamma, \operatorname{Aut}(X))$ is open in $\operatorname{Hom}(\Gamma, \operatorname{Aut}(X))$ and consists of Schottky representations of $\Gamma$ in $\operatorname{Aut}(X)$ with the same fundamental domain as $\Gamma$.

\section{Construction of Uniform Lattices in Rank 2}

Let $X$ be a bihomogeneous, bipartite tree. Let $G \leq \operatorname{Aut}(X)$ be a closed subgroup with a Tits system $(G, B, N)$. Examples of such subgroups are:

(a) $G=K$-rank 1 simple algebraic group over a non-archimedean local field $K$.

(b) $G=$ group of a rank 2 Kac-Moody algebra over a finite field $k$ as in Sec. 6 .

Theorem $11.1([\mathbf{1 8},(\mathbf{2 . 1})])$. Let $X$ be any locally finite bihomogeneous bipartite tree. Let $G \leq \operatorname{Aut}(X)$ be an edge-transitive closed subgroup. Then $G$ contains hyperbolic elements. Moreover, there is a uniform Schottky group $\Gamma \leq G$.

Proof. Lubotzky's stratgey is to first construct a Schottky group in $\operatorname{Aut}(X)$ with finite fundamental domain, and then to check that such a construction can be made within $G$. 
Choose an edge $e \in E X$, the "standard simplex", and label $\partial_{0} e=P_{1}$ and $\partial_{1} e=$ $P_{2}$. Let $P_{2}^{1}, \ldots P_{2}^{r_{2}}$, be the neighbors of $P_{1}$ other than $P_{2}$. For every $i=1, \ldots, r_{2}$, let $P_{1}^{i 1}, \ldots, P_{1}^{i r_{1}}$ be the neighbors of $P_{2}^{i}$, other than $P_{1}$. Similarly, Let $P_{1}^{1} \cdots P_{1}^{r_{1}}$ be the neighbors of $P_{2}$ other than $P_{1}$. For every $i=1, \ldots, r_{1}$, let $P_{2}^{i 1}, \ldots, P_{2}^{i r_{2}}$ be the neighbors of $P_{1}^{i}$, other than $P_{2}$ :

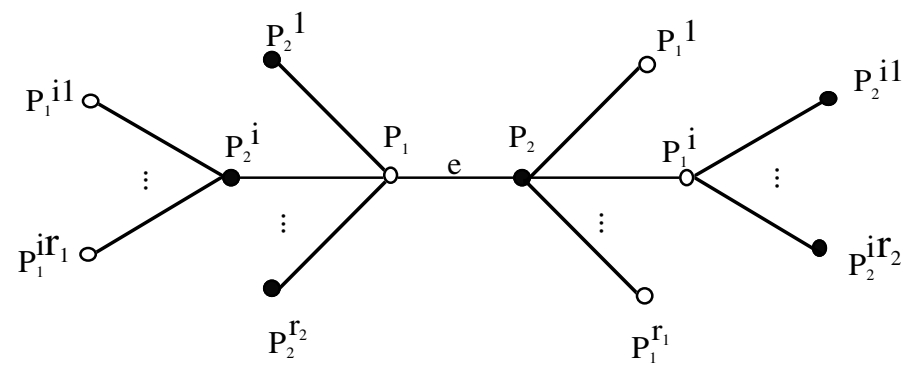

Then $P_{1}$ has $r_{2} \cdot r_{1}$ "grandchildren" $\left\{P_{1}^{i j} \mid i=1, \ldots, r_{2}, j=1, \ldots, r_{1}\right\}$, and $P_{2}$ has $r_{1} \cdot r_{2}$ "grandchildren" $\left\{P_{2}^{i j} \mid i=1, \ldots, r_{1}, j=1, \ldots, r_{2}\right\}$. Choose a one to one correspondence between them, say $P_{1}^{i j}$ corresponds to $P_{2}^{j i}$. Let $\gamma_{i j}$ be an automorphism of $X$ which satisfies:

$$
\gamma_{i j}\left(P_{1}^{i j}\right)=P_{1}^{j} \quad \text { and } \quad \gamma_{i j}\left(P_{2}^{i}\right)=P_{2}^{j i} .
$$

Since $d\left(P_{1}^{i j}, P_{1}^{j}\right)=d\left(P_{2}^{i}, P_{2}^{j i}\right)=4$, Lemma 10.1 implies that $\gamma_{i j}$ is hyperbolic, the axis of $\gamma_{i j}$ passes through

$$
P_{1}^{i j}, P_{2}^{i}, P_{1}, P_{2}, P_{1}^{j}, P_{2}^{j i},
$$

and the amplitude of $\gamma_{i j}$ is $m\left(\gamma_{i j}\right)=4$. By labelling the axis $L\left(\gamma_{i j}\right)$ of $\gamma_{i j}$ such that $x_{0}=P_{1}^{i j}$, we can see easily that the $2 r_{2} r_{1}$ sets $A\left(\gamma_{i j}\right)$ and $B\left(\gamma_{i j}\right)$ are all disjoint.

By Theorem 10.1 the group $\Gamma$ generated by $\left\{\gamma_{i j} \mid i=1, \ldots, r_{2}, j=1, \ldots, r_{1}\right\}$ is therefore a free discrete group on $r_{2} r_{1}$ generators, and a fundamental domain for $\Gamma$ is the set

$$
\left\{P_{1}, P_{2}, P_{1}^{j}, P_{2}^{i} \mid i=1, \ldots, r_{2}, j=1, \ldots, r_{1}\right\}
$$

with $r_{2}+r_{1}+2$ vertices.

Since $G$ acts transitively on the edges of $X$, the elements $\gamma_{i j}$ can be found inside the group $G$. This shows that $G$ contains hyperbolic elements. Therefore, there exists $\gamma_{i j} \in G$ which takes the edge $\left\{P_{1}^{i j}, P_{2}^{i}\right\}$ to the edge $\left\{P_{1}^{j}, P_{2}^{j i}\right\}$. The element $\gamma_{i j} \in G$ necessarily takes $P_{1}^{i j}$ to $P_{1}^{j}$ since $X$ is bihomogeneous and bipartite, and both vertices are of the same type, and similarly, $\gamma_{i j} \in G$ takes $P_{2}^{i}$ to $P_{2}^{j i}$ since both vertices are of the same type. The group $\Gamma$ is a (free) uniform Schottky subgroup of $G$.

Corollary 11.1. Let $G$ be a $K$-rank 1 simple algebraic group over a nonarchimedean local field $K$ or a group of a rank 2 Kac-Moody algebra over a finite field $k$. Then there is a (free) uniform Schottky group $\Gamma \leq G$. 


\section{Deformations of Uniform Lattices in Rank 2}

Using the notation in the previous section, and under the hypotheses of Theorem 11.1, let $\Gamma=\left\langle\gamma_{i j} \mid i=1, \ldots, r_{1}, j=1, \ldots, r_{2}\right\rangle$ be free of rank $r=r_{1} r_{2}$. We apply Theorem 11.1 to the Kac-Moody group of rank 2, as in Sec. 9. For our Kac-Moody group $G$, we have $r_{1}=|k|=r_{2}$. Let $\rho_{0}$ be a faithful representation:

$$
\begin{gathered}
\rho_{0}: \Gamma \longrightarrow G \\
\gamma_{i j} \mapsto g_{i j} .
\end{gathered}
$$

We set

$$
\operatorname{Hom}_{0}(\Gamma, G):=\operatorname{Hom}_{0}(\Gamma, \operatorname{Aut}(X)) \cap \operatorname{Hom}(\Gamma, G) .
$$

Then $\operatorname{Hom}_{0}(\Gamma, G)$ is an open neighborhood of $\rho_{0}$ in $\operatorname{Hom}(\Gamma, G)$ consisting of Schottky representations of $\Gamma$ with the same fundamental domain as $\Gamma$.

Relative to the fundamental domain $F$ for $\Gamma$ as in the proof of Theorem 11.1, and relative to the coordinate system for $X$ as in Fig. 9.1, we can determine the structure of $\operatorname{Hom}_{0}(\Gamma, G)$. Choose the distinguished edge $e$ in the proof of Theorem 11.1 to be the standard simplex. Using the notation in the proof of Theorem 11.1, let

$$
G_{i j}=\left\{\gamma \in G \mid \gamma\left(P_{1}^{i j}, P_{2}^{i}\right)=\left(P_{1}^{j}, P_{2}^{j i}\right)\right\} .
$$

Then relative to the fundamental domain $F$ for $\Gamma$ as in the proof of Theorem 11.1, and relative to the coordinate system for $X$ as in Fig. 9.1,for $G$, we have:

$$
G_{i j}=\chi_{2}\left(s_{j}\right) w_{2} \chi_{1}\left(s_{i}\right) w_{1} B w_{2}^{-1} \chi_{2}\left(s_{j}\right)^{-1} w_{1}^{-1} \chi_{1}\left(s_{i}\right)^{-1},
$$

where $s_{i}, s_{j} \in k, B$ is group of the $(B, N)$ pair as in Theorem 6.1 , and $w_{1}, w_{2}$ are Weyl group generators. Moreover, relative to the fundamental domain $F$ for $\Gamma$ as in the proof of Theorem 11.1, we have

$$
\operatorname{Hom}_{0}(\Gamma, G)=\prod_{i=1, \ldots, r_{1}, j=1, \ldots, r_{2}} G_{i j},
$$

where $r_{1}=|k|=r_{2}$. Thus any choice of

$$
\left(b_{1}, \ldots, b_{r}\right) \in B \times \cdots \times B,
$$

$\left(r=|k|^{2}\right.$ factors) gives rise to generating set for a free uniform lattice of rank $r=|k|^{2}$ in $G$ with the same fundamental domain as $\Gamma$. We have

\section{Conjecture 12.1.}

$$
\operatorname{Hom}_{0}(\Gamma, G) / \text { conjugation }
$$

is uncountable.

Conjecture 12.1 will follow from the following:

Conjecture 12.2. The orbits for the conjugation action on $\operatorname{Hom}_{0}(\Gamma, G)$ are nowhere dense. 
(cf. [7, Conjecture 3.3]). The following result shows that the uniform Schottky groups we have constructed are all conjugate in $\operatorname{Aut}(X)$.

Proposition 12.1. Let $X$ be a locally finite tree, let $\rho_{0}: \Gamma \longrightarrow \operatorname{Aut}(X)$ and $\rho_{1}: \Gamma \longrightarrow \operatorname{Aut}(X)$ be faithful free representations of a group $\Gamma$ in $\operatorname{Aut}(X)$ (that is, $\rho_{0}(\Gamma)$ and $\rho_{1}(\Gamma)$ act freely on $\left.X\right)$. If there is an isomorphism of graphs

$$
h: A_{0}=\rho_{0}(\Gamma) \backslash X \stackrel{\cong}{\longrightarrow} A_{1}=\rho_{1}(\Gamma) \backslash X,
$$

then $\rho_{0}(\Gamma)$ and $\rho_{1}(\Gamma)$ are conjugate in $\operatorname{Aut}(X)$.

Proof. Since $\rho_{0}(\Gamma)$ and $\rho_{1}(\Gamma)$ act freely on $X$, we have

$$
\rho_{0}(\Gamma)=\pi_{1}\left(A_{0}, *\right), \quad \rho_{1}(\Gamma)=\pi_{1}\left(A_{1}, *\right),
$$

both free groups. Since

$$
h: A_{0} \stackrel{\cong}{\longrightarrow} A_{1},
$$

there is a lift

$$
f=\tilde{h}: X \stackrel{\cong}{\longrightarrow} X
$$

with the property

$$
f\left(\rho_{0}(\gamma) x\right)=\rho_{1}(\gamma) f(x)
$$

for $x \in X$, that is

$$
\rho_{0}(\cdot)=f^{-1} \circ \rho_{1}(\cdot) \circ f
$$

for $f \in \operatorname{Aut}(X)$.

Corollary 12.1. Let $\Gamma_{1}$ and $\Gamma_{2} \leq G$ be uniform Schottky subgroups with the same fundamental domain. Let $X$ be the Bruhat-Tits tree of $G$. Then $\Gamma_{1}$ and $\Gamma_{2}$ are conjugate in $\operatorname{Aut}(X)$.

\section{Uniform Commensurability in Rank 2}

Let $X$ be a locally finite tree. A group $\Gamma$ is a uniform lattice in $\operatorname{Aut}(X)$ if and only if $\Gamma$ is finitely generated and virtually free [1, Theorem 8.4]. Furthermore, results of Bass-Kulkarni and Liu give us certain commensurability theorems for uniform lattices our in rank 2 Kac-Moody group. We have the following

Theorem 13.1 ([3, Corollary 4.8]). Let $X$ be a tree that covers a finite connected graph. If $\Gamma_{1}, \Gamma_{2}$ are uniform lattices in $\operatorname{Aut}(X)$, then $g \Gamma_{1} g^{-1}$ and $\Gamma_{2}$ are commensurable for some $g \in \operatorname{Aut}(X)$.

Let $\Gamma$ be a uniform lattice in $\operatorname{Aut}(X)$. Let

$$
C_{\operatorname{Aut}(X)}(\Gamma)=\left\{g \in \operatorname{Aut}(X) \mid g \Gamma g^{-1} \text { and } \Gamma \text { are commensurable }\right\}
$$


be the commensurability group of $\Gamma$ in $\operatorname{Aut}(X)$. A corollary to Theorem 13.1 is the following:

Corollary $13.1([3])$. If $\Gamma_{1}, \Gamma_{2}$ are uniform lattices in $\operatorname{Aut}(X)$, then their commensurability groups $C_{\mathrm{Aut}(X)}\left(\Gamma_{1}\right)$ and $C_{\mathrm{Aut}(X)}\left(\Gamma_{2}\right)$ are conjugate in $\operatorname{Aut}(X)$.

We also have the following:

Theorem $13.2([\mathbf{1 9}])$. For any uniform lattice $\Gamma$, in $\operatorname{Aut}(X), C_{\mathrm{Aut}(X)}(\Gamma)$ is dense in $\operatorname{Aut}(X)$.

Let $G_{0}=G_{0}\left(\mathbb{F}_{p}\right)$ be the group of a rank 2 Kac-Moody algebra over the finite field $\mathbb{F}_{p}$. Let $G_{1}=S L_{2}\left(\mathbb{Q}_{p}\right), G_{2}=S L_{2}\left(\mathbb{F}_{p}\left(\left(t^{-1}\right)\right)\right)$. Then $G_{0}, G_{1}$, and $G_{2}$ have Bruhat-Tits tree $X_{p+1}$. Theorem 13.1 implies:

(1) Any two uniform lattices in the same group $\left(G_{0}, G_{1}\right.$, or $\left.G_{2}\right)$ are commensurable to each other (up to conjugation in $\operatorname{Aut}\left(X_{p+1}\right)$ ).

(2) Any two uniform lattices in different groups $\left(G_{0}, G_{1}\right.$, or $\left.G_{2}\right)$ are commensurable to each other (up to conjugation in $\operatorname{Aut}\left(X_{p+1}\right)$ ).

(3) Any uniform lattice in $G_{0}, G_{1}$, or $G_{2}$ is commensurable to a uniform lattice in $\operatorname{Aut}\left(X_{p+1}\right)$ (up to conjugation in $\operatorname{Aut}\left(X_{p+1}\right)$ ).

Since any uniform lattice in $G_{0}, G_{1}$, or $G_{2}$ is a uniform lattice in $\operatorname{Aut}\left(X_{p+1}\right)$, we have:

(5) If $\Gamma_{1}$ and $\Gamma_{2}$ are uniform lattices in $G_{0}, G_{1}$, or $G_{2}$, then $C_{\mathrm{Aut}\left(X_{p+1}\right)}\left(\Gamma_{1}\right)$ and $C_{\mathrm{Aut}\left(X_{p+1}\right)}\left(\Gamma_{2}\right)$ are conjugate in $\operatorname{Aut}\left(X_{p+1}\right)$.

Theorem (13.3) implies

(6) For any uniform lattice $\Gamma$ in $G_{0}, G_{1}$, or $G_{2}, C_{\operatorname{Aut}\left(X_{p+1}\right)}(\Gamma)$ is dense in $\operatorname{Aut}\left(X_{p+1}\right)$.

We pose the following

Question 13.1. Let $\Gamma$ be a uniform lattice in $G_{0}$. Is $C_{G_{0}}(\Gamma)$ dense in $G_{0}$ ?

\section{A Spherical $B N$-Pair in Rank 2}

Using the notation of Sec. 9, we let $\mathcal{A}$ be any apartment in $X$. Also fix a "base edge" $\sigma(\mathcal{A})$ of $\mathcal{A}$, and $g \in G$, such that

$$
g \cdot \sigma(\mathcal{A})=\sigma_{B}
$$

(so $\left.\sigma(\mathcal{A})=\sigma_{g^{-1} B g}\right)$. Replacing $\mathcal{A}$ by $g \cdot \mathcal{A}$, we may in fact assume

$$
\sigma(\mathcal{A})=\sigma_{B} .
$$

For an apartment $\mathcal{A}$ with base edge $\sigma(\mathcal{A})$, let $\sigma_{1}(\mathcal{A}), \sigma_{2}(\mathcal{A})$ be the two vertices of $\sigma(\mathcal{A})$. Interchanging $\sigma_{1}(\mathcal{A}), \sigma_{2}(\mathcal{A})$ if necessary, we may assume that for some $g \in G$ 
34 L. Carbone \&3 H. Garland

(we define $g(H)=g H g^{-1}$, for a subset $H \subseteq G$ )

$$
\begin{gathered}
\sigma_{1}(\mathcal{A})=\sigma_{g\left(P_{1}\right)}, \\
\sigma_{2}(\mathcal{A})=\sigma_{g\left(P_{2}\right)}, \\
\sigma(\mathcal{A})=\sigma_{g(B)} .
\end{gathered}
$$

We let $\mathcal{A}^{+}$be the subcomplex of $\mathcal{A}$ consisting of the ray joined to $\sigma(\mathcal{A})$ at $\sigma_{2}(\mathcal{A})$, and not containing $\sigma(\mathcal{A})$. We let $\mathcal{A}^{-}$be the subcomplex of $\mathcal{A}$ consisting of $\mathcal{A}-$ $\left(\mathcal{A}^{+} \cup \sigma(\mathcal{A})\right)$. For the standard apartment $\mathcal{A}_{0}$ we take $\sigma\left(\mathcal{A}_{0}\right)=\sigma_{B}$, and then have $\mathcal{A}_{0}^{ \pm}$defined. Hence

$$
\mathcal{A}=\mathcal{A}^{+} \sqcup \mathcal{A}^{-} \sqcup \sigma(\mathcal{A}) .
$$

Assume $(\mathcal{A}, \sigma(\mathcal{A}))$ satisfies $(14.2)$, so $\sigma_{1}(\mathcal{A})=\sigma_{P_{1}}, \sigma_{2}(\mathcal{A})=\sigma_{P_{2}}$. Then we have

Lemma 14.1. We can find elements $b_{1}, b_{2} \in U$ such that

$$
\begin{gathered}
b_{1} \cdot \mathcal{A}^{+}=\mathcal{A}_{0}^{+}, \\
b_{2} b_{1} \cdot \mathcal{A}^{-}=\mathcal{A}_{0}^{-},
\end{gathered}
$$

and furthermore $b_{2}$ stabilizes $\mathcal{A}_{0}^{+}$pointwise.

Proof. For (14.3), we first consider:



where $\tau$ is the (unique) edge of $\mathcal{A}^{+}$with $\sigma_{P_{2}}$ as vertex. Moreover, $\tau$ and $w_{2} \cdot \sigma_{B}$ are two edges with $\sigma_{P_{2}}$ as a vertex, and both distinct from $\sigma_{B}$. We may describe the set of all such edges as follows:

$$
\left\{u \cdot w_{2} \cdot \sigma_{B}\right\}_{u \in U_{\alpha_{2}}},
$$

where $U_{\alpha_{2}}=\left\{\chi_{\alpha_{2}}(s)\right\}_{s \in k}\left(\alpha_{2}\right.$ being the simple root corresponding to $\left.w_{2}\right)$. Hence

$$
\tau=u w_{2} \cdot \sigma_{B}=\sigma_{u w_{2} B w_{2}^{-1} u^{-1}}
$$

for some $u \in U_{\alpha_{2}}$ and, since $u$ stabilizes $\sigma_{B}(u \in B)$, we may assume, replacing $\mathcal{A}^{+}$ by $u^{-1} \mathcal{A}^{+}$, that $\tau=w_{2} \cdot \sigma_{B}$. Let

$$
(\tau=) \tau_{1}, \tau_{2} \ldots
$$


be the edges of $\mathcal{A}^{+}$, going out to $\infty$. Assume inductively, that for some $b \in U \subseteq B$, we have

$$
\begin{aligned}
b \cdot \tau_{1} & =w_{2} \cdot \sigma_{B} \\
b \cdot \tau_{2} & =w_{2} w_{1} \cdot \sigma_{B} \\
& \vdots \\
b \cdot \tau_{k} & =w \cdot \sigma_{B}, w=w_{2} w_{1} \cdots \quad(k \text { factors }), l(w)=k .
\end{aligned}
$$

(We have begun the induction, by proving such a $b$ exists for $\tau_{1}$.) We have

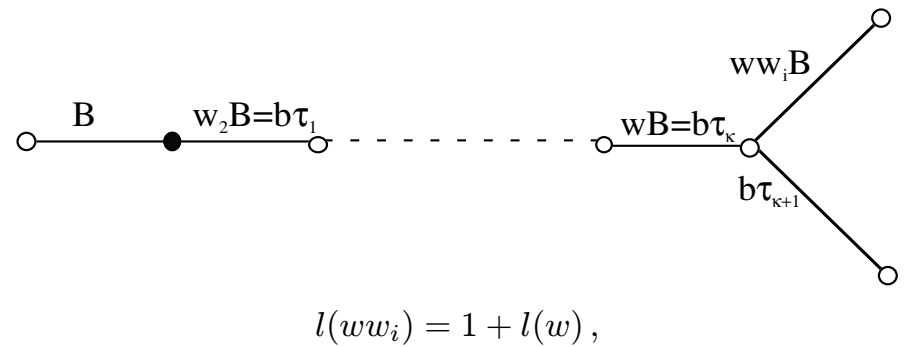

where $i=1$ or 2 . Now

$$
w=w_{2} w_{1} \cdots w_{j}, \quad j \neq i, \quad \text { (reduced expression), }
$$

( $k$ factors). We have, filling in the vertices at the right of the above diagram,

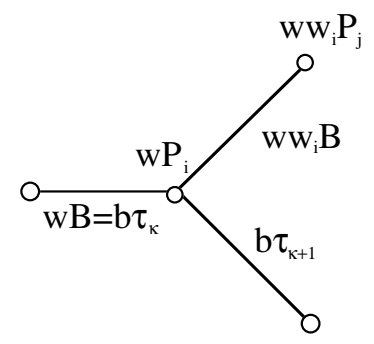

The edges other than $w \cdot \sigma_{B}$, having $w \cdot \sigma_{P_{i}}$ as a vertex are just the $w$-transforms of edges other than $\sigma_{B}$, having $\sigma_{P_{i}}$ as a vertex, and this is just the set:

$$
w \cdot u \cdot w_{i} \cdot \sigma_{B}, \quad u \in U_{\alpha_{i}}
$$

which is the set of all

$$
w u w^{-1} \cdot w w_{i} \sigma_{B}
$$

We claim that

$$
w u w^{-1} \in U \subseteq B
$$

Indeed,

$$
w U_{\alpha_{i}} w^{-1}=U_{w \cdot \alpha_{i}},
$$


36 L. Carbone \& $H$. Garland

and since

$$
l\left(w w_{i}\right)=1+l(w)
$$

$w \cdot \alpha_{i}$ is positive. We have that

$$
b \cdot \tau_{k+1}=u w w_{i} \cdot \sigma_{B}, \quad \text { for some } u \in U_{w \cdot \alpha_{i}},
$$

and

$$
\begin{gathered}
u^{-1} \cdot \sigma_{B}=\sigma_{B}, \\
u^{-1} \cdot w_{2} \cdot \sigma_{B}=w_{2} \cdot \sigma_{B}, \\
\vdots \\
u^{-1} \cdot w \cdot \sigma_{B}=w \cdot \sigma_{B} .
\end{gathered}
$$

Moreover, inductively assume

$$
b \in U_{\beta_{1}} \ldots U_{\beta_{k}}
$$

where

$$
\begin{aligned}
\beta_{1} & =\alpha_{i_{1}}=\alpha_{2}, \\
\beta_{2} & =w_{i_{1}} \cdot \alpha_{i_{2}}, \\
& \vdots \\
\beta_{k} & =w_{i_{1}} \cdots w_{i_{k-1}} \cdot \alpha_{i_{k}} \\
& =w_{i_{1}} \cdots w_{i_{k-1}} \cdot \alpha_{j}, \\
w & =w_{i_{1}} \cdots w_{i_{k}}=w_{2} w_{1} \cdots w_{j} ;
\end{aligned}
$$

then

$$
u^{-1} b \in \prod_{\alpha \in \Delta_{+} \cap w w_{i}\left(\Delta_{-}\right)} U_{\alpha}
$$

In summary, we have proved that there is a sequence of elements

$$
u_{1}, \ldots, u_{k}, \ldots \in U
$$

so that

$$
u_{k} \cdot \tau_{q}=w_{2} w_{1} \cdots w_{j} \cdot \sigma_{B}, \quad q \leq k
$$

( $q$ factors), with

$$
u_{k} \in \prod_{\alpha \in \Delta_{+} \cap w\left(\Delta_{-}\right)} U_{\alpha}, \quad w=w_{1} w_{2} \cdots w_{j} \text { (reduced expression) }
$$

and

$$
u_{k+1}=u u_{k}, \quad u \in U_{w \cdot \alpha_{i}}, i \neq j .
$$

In particular, the sequence $u_{k}$ must then converge to a limit $u$, and we may take $b_{1}=u($ in $(14.3))$. 
We now have (replacing $\mathcal{A}$ by $\left.b_{j} \mathcal{A}\right)$ )

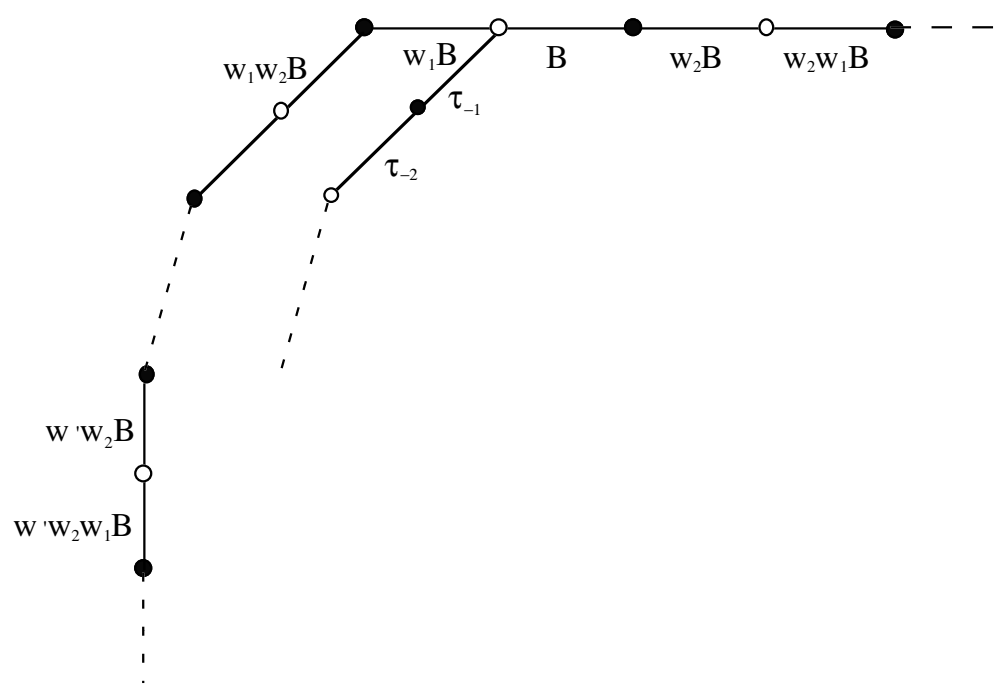

where $w^{\prime}=w_{1} w_{2} \cdots w_{1}$, and we assume now (as we may) that

$$
\tau_{q}=w_{2} w_{1} \cdots \sigma_{B}
$$

$q$ factors, $q=1,2, \ldots$

We now let $\tau_{-1}, \tau_{-2}, \ldots$ be the edges of $\mathcal{A}^{-}$going out to $\infty$, and we let

$$
w(r)=w_{1} w_{2} \cdots,
$$

( $r$ factors) and we manufacture a sequence

$$
\bar{u}_{r} \in \prod_{\alpha \in \Delta_{+} \cap w(r) \Delta_{-}} U_{\alpha}
$$

as before. But

$$
\Delta_{+} \cap w(r) \Delta_{-}=\left\{\alpha_{1}, w_{1} \cdot \alpha_{2}, \ldots\right\},
$$

and each $U_{\alpha}, \alpha \in \Delta_{+} \cap w(r) \Delta_{-}$, leaves each simplex of $\mathcal{A}_{0}^{+}$fixed, and we may take $b_{2}$ to be the limit of the $\bar{u}_{r}$.

Corollary 14.1. $G$ acts doubly transitively on the ends of $X$.

Corollary 14.2. Let $\mathcal{B}^{ \pm}$be the stabilizer of the end $\mathcal{E}_{0}^{ \pm}$determined by $\mathcal{A}_{0}^{ \pm}$; then $\left(\mathcal{B}=\mathcal{B}^{+}\right)$we have

$$
G=\mathcal{B} \sqcup \mathcal{B} w_{1} \mathcal{B}=\mathcal{B} \sqcup \mathcal{B} w_{2} \mathcal{B}
$$

Proof. We note that $w_{1} \cdot\left(\sigma_{B} \cup \mathcal{A}_{0}^{+}\right)=\mathcal{A}_{0}^{-}$. We let $g \in G$, and consider $g \cdot \mathcal{A}_{0}$; then $g \cdot \mathcal{A}_{0}^{+}, g \cdot \mathcal{A}_{0}^{-}$determine two ends $g \cdot \mathcal{E}_{0}^{ \pm}$of $X$ and by Corollary 14.1, if $g \notin \mathcal{B}$, there exists $b \in G$ such that $b \cdot \mathcal{E}_{0}^{+}=\mathcal{E}_{0}^{+}$and $b \cdot g \mathcal{E}_{0}^{+}=\mathcal{E}_{0}^{-}$; then $b \in \mathcal{B}$ and

$$
w_{1} b \cdot g \mathcal{E}_{0}^{+}=w_{1} \cdot \mathcal{E}_{0}^{-}=\mathcal{E}_{0}^{+}
$$


38 L. Carbone \&3 H. Garland

Hence

$$
w_{1} b g=b^{\prime} \in \mathcal{B}
$$

or

$$
g=b^{-1} w_{1} b^{\prime}
$$

We are left with the question of describing $\mathcal{B}$ explicitly. We do this as follows: we let

$$
\begin{aligned}
\mathcal{B}_{\mathcal{I}} & =\bigcap_{w \in W} w B w^{-1} \subset B, \\
T & =\left\{\left(w_{1} w_{2}\right)^{n}\right\}_{n \in \mathbb{Z}}\left(\text { translations along } \mathcal{A}_{0}\right), \\
\mathcal{U} & =\text { closure of } \mathcal{U}_{0}, \text { where } \\
\mathcal{U}_{0} & =\text { group generated by all } U_{\alpha}, \text { with } \\
\alpha & \in \Phi_{1},
\end{aligned}
$$

where

$$
\Phi_{1}:=\left\{-\alpha_{2},-w_{2} \cdot \alpha_{1},-w_{2} w_{1} \cdot \alpha_{2} \cdots\right\} \cup\left\{\alpha_{1}, w_{1} \cdot \alpha_{2}, w_{1} w_{2} \cdot \alpha_{1} \cdots\right\} .
$$

From the Appendix, we have Fig. A2, for $k>2$ :

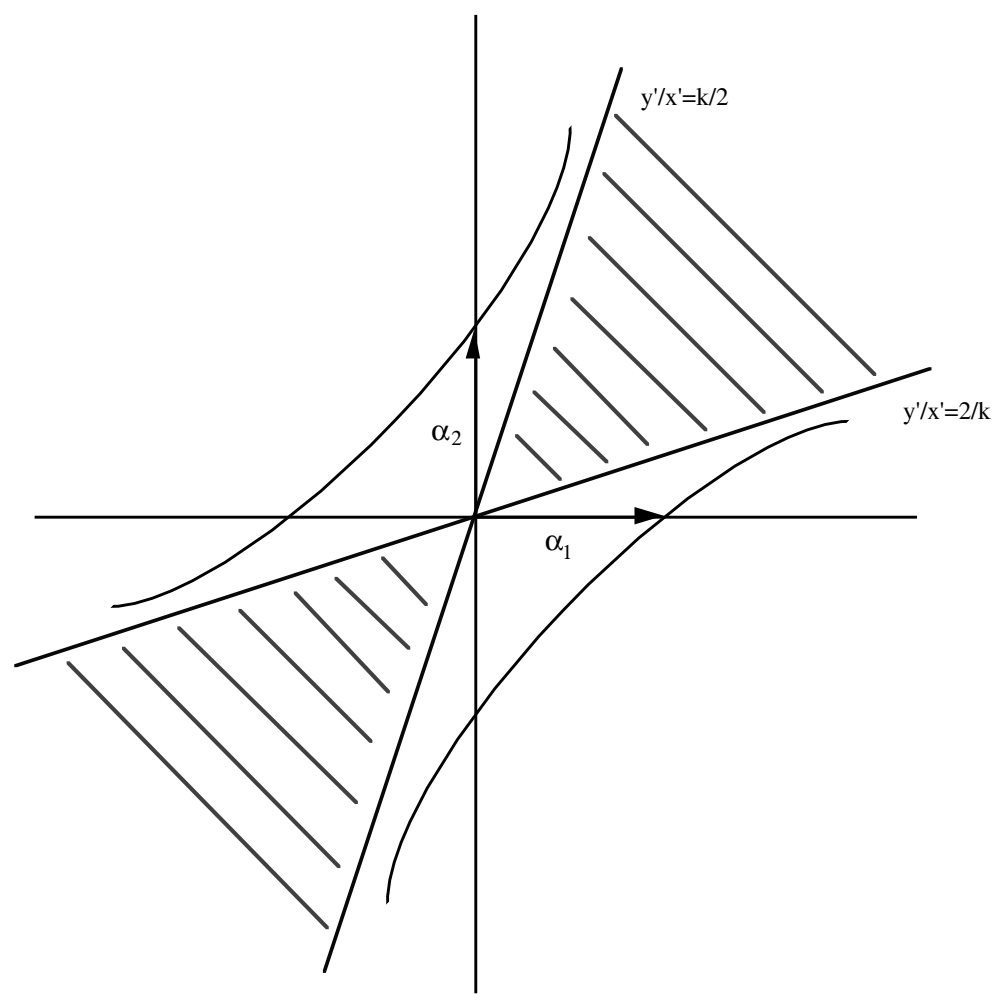


We have that the Weyl roots are distributed along two branches of the hyperbola (pictured above) with $\alpha_{1}$ and $\alpha_{2}$ on two different branches. Since

$$
w_{1} \cdot \alpha_{1}=-\alpha_{1}, \quad w_{2} \cdot \alpha_{2}=-\alpha_{2},
$$

$w_{1}$ and $w_{2}$ must interchange the two branches of the hyperbola.

Hence the Weyl roots in

$$
\Phi_{1}=\left\{-\alpha_{2},-w_{2} \cdot \alpha_{1},-w_{2} w_{1} \cdot \alpha_{2} \cdots\right\} \cup\left\{\alpha_{1}, w_{1} \cdot \alpha_{2}, w_{1} w_{2} \cdot \alpha_{1} \cdots\right\}
$$

are distributed along the lower branch of the hyperbola. The remaining Weyl roots, namely those in

$$
\Phi_{2}:=\left\{-\alpha_{1},-w_{1} \alpha_{2},-w_{1} w_{2} \alpha_{1}, \ldots\right\} \cup\left\{\alpha_{2}, w_{2} \alpha_{1}, w_{2} w_{1} \alpha_{2}, \ldots\right\}
$$

are distributed along the upper branch.

We have

$$
\Phi=\Phi_{1} \cup \Phi_{2}, \quad \text { (disjoint union) } .
$$

Moreover, the imaginary roots lie inside the imaginary cone which coincides with the shaded region between the asymptotes.

Since the sum of any two roots along the lower branch is not on either of the branches, nor in the shaded region between the asymptotes, such a sum cannot be a root.

Lemma 14.2. Let $\alpha, \beta$ be Weyl roots. If $\alpha+\beta$ is not zero or a root, then $\left[U_{\alpha}, U_{\beta}\right]=0$.

Proof. Since $\alpha+\beta$ is not zero or a root, in the Kac-Moody algebra, the corresponding root vectors $x_{\alpha}$ and $x_{\beta}$ commute. Hence any power or divided power of $x_{\alpha}$ commutes with any power or divided power of $x_{\beta}$.

Hence the $U_{\alpha}$ with $\alpha \in \Phi_{i}, i=1$ or $i=2$, must commute among themselves, and the group $\mathcal{U}$ is commutative. We refer the reader to [20] for additional details on hyperbolic root systems, and [21] and [30] for more commutator relations in Kac-Moody groups.

The group $T$ normalizes $\mathcal{U}$ and we have

Theorem 14.1. $\mathcal{B}=\mathcal{B}_{\mathcal{I}} \mathcal{U} T=\mathcal{B}_{\mathcal{I}} T \mathcal{U}=\mathcal{U} T \mathcal{B}_{\mathcal{I}}=\mathcal{U} \mathcal{B}_{\mathcal{I}} T$.

Proof. $g \in \mathcal{B}$ implies that there exist $n, m \in \mathbb{Z}$ such that

$$
g \cdot\left(w_{2} w_{1}\right)^{m}\left(\mathcal{A}_{0}^{+} \cup \sigma_{B}\right)=\left(w_{2} w_{1}\right)^{n}\left(\mathcal{A}_{0}^{+} \cup \sigma_{B}\right) .
$$

We set $t=w_{2} w_{1}$, and note that from the above equality, we have

$$
t^{-n} g t^{m}\left(\mathcal{A}_{0}^{+} \cup \sigma_{B}\right)=\left(\mathcal{A}_{0}^{+} \cup \sigma_{B}\right),
$$

and $t^{-n} g t^{m}$ fixes $\mathcal{A}_{0}^{+} \cup \sigma_{B}$ pointwise. Consider

$$
t^{-n} g t^{m}\left(\mathcal{A}_{0}^{-}\right)
$$


then the argument of Lemma 14.1 shows that there exists $b \in \mathcal{U}$ which stabilizes $\mathcal{A}_{0}^{+} \cup \sigma_{B}$ and satisfies

$$
b t^{-n} g t^{m}\left(\mathcal{A}_{0}^{-}\right)=\left(\mathcal{A}_{0}^{-}\right) .
$$

Then

$$
b t^{-n} g t^{m} \text { stabilizes } \mathcal{A}_{0} \text { pointwise. }
$$

Therefore

$$
b t^{-n} g t^{m}:=b_{\mathcal{I}} \in \mathcal{B}_{\mathcal{I}}
$$

Then

$$
g=t^{n} b^{-1} b_{\mathcal{I}} t^{-m}=\left(t^{n} b^{-1} t^{-n}\right)\left(t^{n} b_{\mathcal{I}} t^{-n}\right) t^{n-m}
$$

where

$$
\begin{aligned}
t^{n} b^{-1} t^{-n} & \in \mathcal{U}, \\
t^{n} b_{\mathcal{I}} t^{-n} & \in \mathcal{B}_{\mathcal{I}}, \\
t^{n-m} & \in T,
\end{aligned}
$$

which completes the proof.

\section{Construction of Non-Uniform Lattices in Rank 2}

In this section we use $k$ to denote the finite field $\mathbb{F}_{q}$. In analogy with [18], we let $\mathcal{U}^{\prime} \subseteq \mathcal{U}$ be the subgroup of $\mathcal{U}$ (as defined in (14.5)) defined as follows:

$$
\mathcal{U}^{\prime}=\text { closure of the group generated by all } U_{\alpha} \text {, with } \alpha \in \Phi_{1}^{+},
$$

where

$$
\Phi_{1}^{+}:=\left\{\alpha_{1}, w_{1} \cdot \alpha_{2}, w_{1} w_{2} \cdot \alpha_{1} \cdots\right\} .
$$

Remark 15.1. The group $\mathcal{U}$ is topologically isomorphic to the additive group of $\mathbb{F}_{q}((t))$, the field of formal Laurent series in $t$ over $\mathbb{F}_{q}$, the group $\mathcal{U}^{\prime}$ is isomorphic to the additive group of the ring of formal power series $\mathbb{F}_{q}[[t]], \mathcal{U}^{\prime}$ is profinite, and equals $\mathcal{U} \cap B$. The group $\mathcal{U}$ (respectively $\mathcal{U}^{\prime}$ ) plays the role of the (additive) subgroup of $S L_{2}\left(\mathbb{F}_{q}\left(\left(t^{-1}\right)\right)\right)$ (respectively $\left.S L_{2}\left(\mathbb{F}_{q}\left[\left[t^{-1}\right]\right]\right)\right)$ of upper triangular unipotent matrices in the proof of A. Lubotzky's Proposition 3.2, (see [18, p. 415]).

Our aim is to construct an infinite family of non-uniform lattices in our rank 2 Kac-Moody group $G$.

In analogy with [18], we let $\Delta$ denote the subgroup of $G$ generated by all $\chi_{\alpha}(s)$, where $\alpha \in \Phi_{1}^{-}$(as defined in Sec. 14) (there is an over-use of " $\Delta$ " here, but the notation is standard, for both the set of roots, and the subgroup $\Delta$ of [18]).

Lemma 15.1. We have

$$
\Delta \cong \bigoplus_{i=1}^{\infty}\left(\mathbb{F}_{q}^{+}\right)_{i}
$$

where $\mathbb{F}_{q}^{+}$denotes the additive group of the finite field $\mathbb{F}_{q}$. 
Hence $\Delta \cong \bigoplus_{i=1}^{\infty}(\mathbb{Z} / p \mathbb{Z})_{i}$. It follows that the group $\Delta$ is isomorphic to the group $\Delta$ of $([18$, Sec. 3$])$, and so $\Delta$ is residually finite.

Lemma 15.2. The group $\Delta$ is a uniform lattice in $\mathcal{U}$.

Proof. The group $\Delta$ is a subgroup of $B^{-}$(as in Sec. 6), which is discrete. We have $\mathcal{U}=\mathcal{U}^{\prime} \cdot \Delta$, and $\mathcal{U}^{\prime}$ is compact.

To construct non-uniform lattices, our strategy, following Lubotzky [18], is to enlarge $\Delta$ to a non-uniform lattice in $G$. We have

$$
\begin{aligned}
G / \mathcal{B} & =(\mathcal{B} \sqcup \mathcal{U} w \mathcal{B}) / \mathcal{B} \\
& \cong\{\infty\} \sqcup \mathcal{U} .
\end{aligned}
$$

The decomposition $G / \mathcal{B} \cong\{\infty\} \sqcup \mathcal{U}$ is analogous to the decomposition of $G / B$ in $[18$, p. 417], for a simple algebraic group $G$ over a non-archimedean local field, and a minimal parabolic subgroup $B$. For example if Lubotzky's $G$ is $=S L_{2}$, then the coset $\mathcal{B} / \mathcal{B}$ in $(15.1)$ corresponds to $\{\infty\}$ in $[18$, p. 417], and the coset $\mathcal{U} w \mathcal{B} / \mathcal{B}$ in (15.1) corresponds to $U \cong \mathbb{F}_{q}\left(\left(t^{-1}\right)\right)$ in [18, p. 417], where $U$ is the unipotent radical of $B$. In Lubotzky's setting, one may identify $\{\infty\} \sqcup \mathbb{F}_{q}\left(\left(t^{-1}\right)\right)$ with $\operatorname{Ends}(X)$ where $X$ is the Bruhat-Tits tree of $G$, and

$$
\operatorname{Ends}(X)=\partial X=\{\text { equivalence classes of semi-infinite paths in } X\},
$$

where two such paths are equivalent if their intersection is infinite. Here we also identify $\{\infty\} \sqcup \mathcal{U}$ in (15.1) with $\operatorname{Ends}(X)$, where $X$ is the Bruhat-Tits tree of our Kac-Moody group $G$.

We remark that

$$
\Delta \subseteq \mathcal{U} \subseteq \mathcal{B}=\operatorname{Stab}_{G}(\infty),
$$

where $\mathcal{B}$ is the stabilizer of the end $\infty$ represented by the positive half ray (denoted by $\mathcal{A}_{0, \infty}^{+}$) of the standard apartment $\mathcal{A}_{0}$ (as in Sec. 9). Hence $\Delta$ fixes $\infty$, but no other end of $X$. Let $\mathcal{A}_{0, \infty}^{+}$have vertex sequence $\left(x_{0}, x_{1}, x_{2}, \ldots\right)$ corresponding to the cosets $\left(P_{1}, P_{2}, w_{2} P_{1}, \ldots\right)$ as in Fig. 15.1.

We let $F \subseteq X$ consist of the union of all bi-infinite lines containing $x_{0}$ (notation of Fig. 15.1) and having $\infty$ as one end ( $F$ is shown in Fig. 15.1, in the case $|k|=2$ ). Then any semi-infinite ray in $F$ with origin $x_{0}$ and end different from $\infty$ can be "collapsed" to the standard ray (with end $-\infty$ determined by $\mathcal{A}_{0}^{-}$) by an element of $\mathcal{U}^{\prime} \subseteq \operatorname{Stab}_{G}\left(x_{0}\right)$, and conversely, the orbit of the end $-\infty$ under $\mathcal{U}^{\prime}$ corresponds to semi-infinite rays in $F$ with origin $x_{0}$, and end different from $\infty$.

We will now prove

$$
\Delta F=X
$$

Let $x$ be any vertex in $X$, and let $\mathcal{A} \subseteq X$ be an apartment such that:

$$
x \text { is a vertex of } \mathcal{A} \text {. }
$$




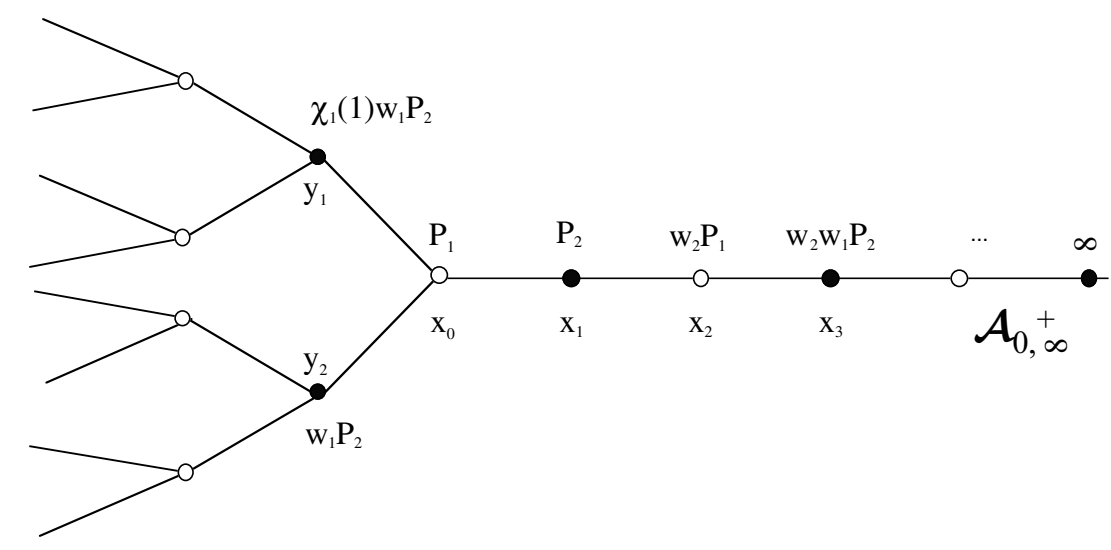

Fig. 15.1. For $|k|=2$, the diagram of $F$, a fundamental domain of $\Delta$ on $X$.

and

$\mathcal{A} \cap \mathcal{A}_{0}$ contains all simplices of $\mathcal{A}_{0}$ to the right of some fixed $x_{i}$

(see Fig. 15.1).

Then $\mathcal{A}$ determines two ends, $\xi, \infty$, where $\xi$ corresponds to some element of $\mathcal{U}$. We can find $\delta \in \Delta$ such that $\delta \cdot \xi \in \mathcal{U}^{\prime}$, as remarked in the proof of Lemma 15.2. But $\delta$ leaves $\infty$ fixed, and so $\delta \cdot \mathcal{A} \subseteq F$. In particular $\delta x \in F$, which proves (15.1).

We quote [18, Proposition 1.10], which we shall use to prove Theorem 15.1.

Proposition 15.1 ([18, (1.10)]). Let $\Gamma_{1}, \ldots, \Gamma_{s}$ be discrete subgroups of $\operatorname{Aut}(X)$ and $x_{0} \in V X$ such that $x_{0}$ is fixed by no non-trivial element of any of the $\Gamma_{i}^{\prime} s$. Let $D_{i}$ be a fundamental domain for $\Gamma_{i}$ containing $x_{0}$, and

$$
E_{i}=E\left(D_{i}\right):=\left\{x \in D_{i} \mid\left(\Gamma_{i}\right)_{x}=\{1\}\right\} .
$$

Assume $E_{i} \supseteq X-E_{j}$ for every pair $i \neq j \in\{1, \ldots, s\}$ and that $\gamma\left(E_{i}\right) \cap E_{i}=\varnothing$, for every $1 \neq \gamma \in \Gamma_{i}$. Let $\Gamma$ be the group generated by $\Gamma_{1}, \ldots, \Gamma_{s}$. Then

(i) $\Gamma$ is discrete.

(ii) $\Gamma$ is the free product of $\Gamma_{1}, \ldots, \Gamma_{s}$.

(iii) $D_{0}=\bigcap_{i=1}^{s} D\left(\Gamma_{i}, x_{0}\right)$ contains the Dirichlet fundamental domain $D\left(\Gamma, x_{0}\right)$ for $\Gamma$ where

$$
D\left(\Gamma, x_{0}\right):=\left\{x \in V X \mid d\left(x, x_{0}\right) \leq d\left(\gamma x, x_{0}\right) \quad \text { for every } \gamma \in \Gamma\right\} .
$$

Theorem 15.1. Using the notation in Fig. 15.1, let $y_{1}, \ldots, y_{q}$ be the neighbours of $x_{0}$ other than $x_{1}$, where $q=|k|$. Choose $g_{0}=i d, g_{1}, \ldots, g_{q}$ in $P_{1} / B=$ $\operatorname{Stab}_{G}\left(x_{0}\right) / \operatorname{Stab}_{G}\left(x_{0}, x_{1}\right)$ subject to:

$$
g_{0}=i d, \quad g_{1}\left(x_{1}\right)=y_{1}, \quad g_{2}\left(x_{1}\right)=y_{2}, \ldots, g_{q}\left(x_{1}\right)=y_{q},
$$


and set $\Delta_{i}=g_{i} \Delta g_{i}^{-1}, i=0, \ldots, q$. Let $\Gamma$ be the group generated by $\Delta_{0}, \ldots, \Delta_{q}$. Then

(1) $\Gamma$ is discrete,

(2) $\Gamma$ equals the free product $\Delta_{0} * \cdots * \Delta_{q}$, and

(3) $\Gamma$ is a non-uniform lattice in $G$.

Proof. We apply [18, Proposition 1.10] (cf. [18, Proposition 3.2]). First we show that

$$
\Delta_{0} \cap P_{1}=\{1\} .
$$

It then follows that the vertex $x_{0}$ is fixed by no non-trivial element of $\Delta_{i}, i=$ $0, \ldots, q$. To prove (15.4), we show:

$$
w_{1} \Delta_{0} w_{1}^{-1} \subseteq U^{-}
$$

and

$$
P_{1} \cap U^{-}=U_{-\alpha_{1}} .
$$

Assertion (15.5a) follows at once from the definition of $\Delta_{0}$. For (15.5b), if $\xi \in P_{1}$ is in $U^{-}$, it is lower triangular unipotent with respect to a coherently ordered basis. Let

$$
\begin{aligned}
& v_{\lambda}=\text { a highest weight vector, } \\
& L_{1}=\text { the subgroup generated by } \chi_{ \pm \alpha_{1}}(s), s \in k .
\end{aligned}
$$

Let $W$ be the $L_{1}$-submodule of $V_{k}^{\lambda}$ generated by $v_{\lambda}$; then $W$ is spanned by vectors

$$
v_{\lambda}, v_{\lambda-\alpha_{1}}, \ldots, v_{\lambda-m \alpha_{1}}=w_{1} v_{\lambda}
$$

where $v_{\lambda-q \alpha_{1}} \in V_{\lambda-q \alpha_{1}}^{\lambda}, q=0,1, \ldots, m$.

Then $W$ is $P_{1}$-invariant, and moreover $P_{1}=L_{1} U_{1}$,

$$
U_{1}=U \cap w_{1} U w_{1}^{-1},
$$

and $U_{1}$ acts trivially on $W$, as follows from the equality $U_{0, w_{i}}=U^{\prime}$ of Sec. 6 . Then we have

$$
\xi=\xi_{L} \xi_{U}, \quad \xi_{L} \in L_{1}, \xi_{U} \in U_{1}
$$

and

$$
\left.\xi_{L}\right|_{W}=\left.\xi\right|_{W}, \quad \text { so } \xi_{L} \text { is lower triangular, unipotent. }
$$

Hence $\xi_{L} \in U_{-\alpha_{1}}$, and thus

$$
\xi_{U}=\xi_{L}^{-1} \xi
$$


where the right-hand side is lower triangular unipotent, and the left-hand side is upper triangular unipotent. Thus $\xi_{U}=1$, and so $\xi=\xi_{L} \in U_{-\alpha_{1}}$, and we have proven $(15.5 \mathrm{~b})$.

It follows from (15.5a) and (15.5b) that

$$
w_{1} \Delta_{0} \cap P_{1} w_{1}^{-1}=w_{1} \Delta_{0} w_{1}^{-1} \cap P_{1} \subseteq U^{-} \cap P_{1} \subseteq U_{-\alpha_{1}},
$$

and so

$$
\Delta_{0} \cap P_{1} \subseteq U_{\alpha_{1}}
$$

But

$$
\Delta_{0} \cap U_{\alpha_{1}}=\{1\},
$$

since $U_{\alpha_{1}}$ is upper triangular with 1's on the diagonal, and $\Delta_{0}$ is lower triangular with 1's on the diagonal. Thus $\Delta_{0} \cap P_{1}=\{1\}$ and we have proven (15.4).

Since $\Delta$ fixes the unique end $\infty$ of $X$ but not $x_{0}$, we have

$$
\begin{aligned}
E_{0}=E_{0}(\Delta): & =\left\{x \in F \mid \Delta_{x}=\{1\}\right\} \\
& =\left\{x \in F \mid d\left(x, x_{0}\right) \leq d\left(x, x_{1}\right)\right\} .
\end{aligned}
$$

It follows that

$$
\gamma E_{0} \cap E_{0}=\varnothing, \quad 1 \neq \gamma \in \Delta .
$$

For $i=1, \ldots, q$, we set

$$
E_{i}=E_{i}(\Delta):=\left\{x \in F \mid\left(\Delta_{i}\right)_{x}=\{1\}\right\} .
$$

Then each subtreee $E_{i} \subseteq X$ is a rotation in $X$ of the subtree $E_{0}$ around the vertex $x_{0}$. It follows that

$$
\gamma E_{i} \cap E_{i}=\varnothing, \quad 1 \neq \gamma \in \Delta_{i}, i=1, \ldots, q .
$$

It is also easy to verify that

$$
X-E_{j} \subseteq E_{i}, \quad i \neq j,
$$

$i=1, \ldots, q$.

It follows from [18, Proposition 1.10] that $\Gamma$ is discrete, $\Gamma$ equals the free product $\Delta_{0} * \cdots * \Delta_{q}$, and that a fundamental domain $Y$ for $\Gamma$ on $X$ is

$$
Y=\bigcap_{i=0}^{q} g_{i}(F)=\bigcup_{i=0}^{q} g_{i}\left(\mathcal{A}_{0, \infty}^{+}\right),
$$

depicted in Fig. 15.2: 


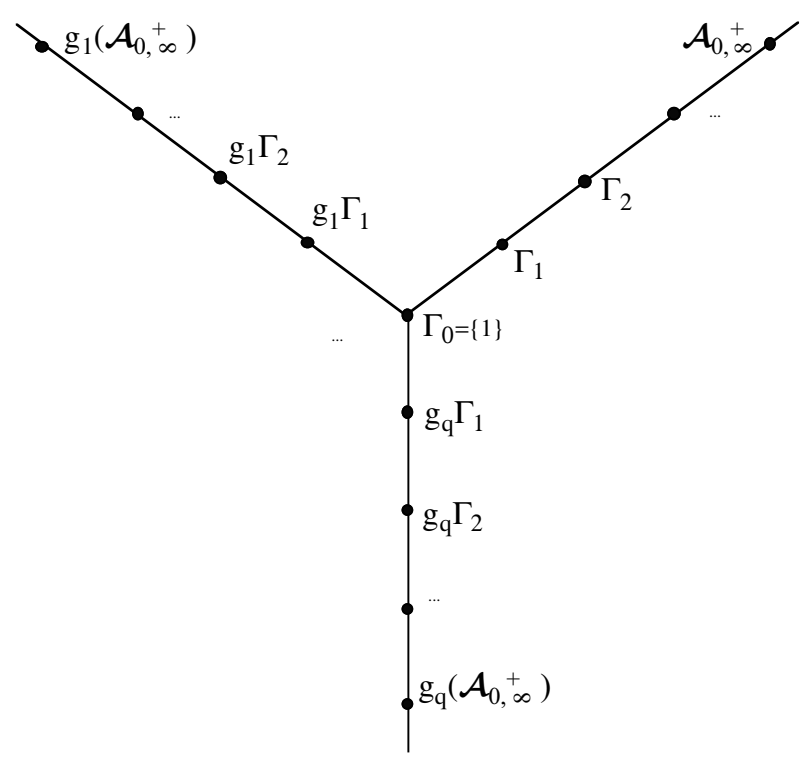

Fig. 15.2.

To compute the isotropy groups of vertices and edges in $Y$, we recall (Sec. 9) that

$$
E \mathcal{A}_{0, \infty}^{+}=\left\{B, w_{2} B, w_{2} w_{1} B, \ldots\right\} .
$$

For $w \in W$, let $\Gamma_{w}$ denote the isotropy group of the coset $w B$ :

$$
\begin{aligned}
\Gamma_{w} & =\{\gamma \in \Gamma \mid \gamma w B=w B\} \\
& =\Gamma \cap w B w^{-1} .
\end{aligned}
$$

Then $\Gamma_{w}$ is a discrete subgroup of the compact group $w B w^{-1}$ and hence is finite.

We have

$$
\left|\Gamma \cap w B w^{-1}\right| \geq\left|\Delta_{0} \cap w B w^{-1}\right| \geq q^{l(w)}
$$

(the last inequality following from the arguments of Lemma 8.3), where $l(\cdot)$ is the length function on $W$.

For $n \geq 0$, let $\Gamma_{n}$ be the isotropy group of the vertex $x_{n}$ of the positive half ray $\mathcal{A}_{0, \infty}^{+}=\left(x_{0}, x_{1}, x_{2}, \ldots\right)$ (using notation as in Figs. 15.1 and 15.2). Then for $n \geq 1$, $i=1, \ldots, q$

$$
\Gamma_{n} \cong g_{i}\left(\Gamma_{n}\right)
$$

Applying (15.6) we obtain

$$
\Gamma_{0}=\{1\},
$$


and for $n \geq 1, i=1, \ldots, q$ :

$$
\left|\Gamma_{n}\right|=\left|g_{i}\left(\Gamma_{n}\right)\right| \geq q^{n} .
$$

Thus the covolume of $\Gamma$, suitably normalized, is at most:

$$
(q+1) \sum_{i=0}^{\infty} \frac{1}{q^{i}} .
$$

So $\Gamma$ is a non-uniform lattice in $G$.

Remark 15.2. (1) It follows from Lemma 15.1 that the group $\Gamma$ constructed in Theorem 15.1 is isomorphic to $\Gamma$ of [18, Proposition 3.2].

(2) The group $\Gamma$ constructed in Theorem 15.1 cannot be finitely generated $[3,(0.4)]$.

\section{Deformations of Non-Uniform Lattices in Rank 2}

In this section, we describe how the construction of non-uniform lattices in Sec. 15 is flexible enough to admit an uncountably infinite family of non-uniform lattices. We describe a compact parameter space for this uncountably infinite family. It remains to determine whether non-uniform lattices form uncountably many conjugacy classes in the Kac-Moody group. We conjecture that this is the case (Conjecture 16.1).

\subsection{Cuspidal deformations}

Using the notation of Sec. 15, and Theorem 15.1, we have a non-uniform lattice $\Gamma$ which is the free product

$$
\Gamma=\Delta_{0} * \cdots * \Delta_{q}
$$

By varying the $g_{i}$ over the open set $B /\left(B \cap N_{G}(\Delta)\right)$, where $N_{G}(\Delta)$ denotes the normalizer in $G$ of $\Delta$, for $i=1, \ldots q$, we have an uncountably infinite family of non-uniform lattices here, parametrized by an open set of the form:

$$
\mathcal{P}(\Gamma, G)=B /\left(B \cap N_{G}(\Delta)\right) \times \cdots \times B /\left(B \cap N_{G}(\Delta)\right)
$$

$(q=|k|$ factors $)$.

Lemma 16.1. We have $N_{G}(\Delta) \subseteq \mathcal{B}$.

Proof. Recall (Sec. 15) that $\Delta$ fixes $\infty$ but no other end of $X$. We claim that if $g \Delta g^{-1} \subseteq \Delta \subseteq \mathcal{B}$, then $g \in \mathcal{B}$. Suppose, conversely that $g \notin \mathcal{B}$. If $g \notin \operatorname{Stab}_{G}(\infty)$, then for every $1 \neq \delta \in \Delta$ one has $g \delta g^{-1} \notin \mathcal{B}$. Otherwise $g \delta g^{-1} \in \mathcal{B}$, and has $\infty$ as a fixed point. However, since $\delta$ has $\infty$ as a fixed point, it is the case that $g \delta g^{-1}$ has $g \cdot \infty$ as a fixed point. Since $g \notin \operatorname{Stab}_{G}(\infty), g \cdot \infty \neq \infty$, and so $g \delta g^{-1}$ has two fixed 
points on the boundary. Hence $\delta$ has two fixed points on the boundary, which is a contradiction. Hence $g \in \mathcal{B}$ as asserted.

The question arises as to whether we have uncountably many distinct conjugacy classes of non-uniform lattices in $G$. We have natural maps:

$$
\begin{aligned}
B /\left(B \cap N_{G}(\Delta)\right) \times & \cdots \times B /\left(B \cap N_{G}(\Delta)\right) \\
& \downarrow \\
B /(B \cap \mathcal{B}) \times & \cdots \times B /(B \cap \mathcal{B}) \\
& \downarrow \\
G / \mathcal{B} \times & \cdots \times G / \mathcal{B}, \\
& \cong \\
(\mathcal{U} \sqcup \infty) \times & \cdots \times(\mathcal{U} \sqcup \infty) \\
& \cong \\
\partial X \times & \cdots \partial X .
\end{aligned}
$$

( $q=|k|$ factors). We have the following (cf. [7, Conjecture 2.12]):

Conjecture 16.1. The diagonal action of $G$ on

$$
G / \mathcal{B} \times \cdots \times G / \mathcal{B}
$$

$(|k|$ factors $)$, has the property that every orbit is nowhere dense.

An affirmative answer to Conjecture 16.1 would imply the existence of uncountably many conjugacy classes of non-uniform lattices in $G$. Suppose that $A$ is affine, that is,

$$
A=\left(\begin{array}{cc}
2 & -2 \\
-2 & 2
\end{array}\right) \text {. }
$$

Then $G$ is a central extension, denoted $\widehat{S L_{2}}$

$$
1 \longrightarrow k^{\times} \longrightarrow \widehat{S L_{2}} \longrightarrow S L_{2} \longrightarrow 1
$$

of $S L_{2}$ over a Laurent series field $k((t))$ over the finite field $k$, by the (one dimensional) multiplicative group $k^{\times}$. In this case, our uncountably infinite family of nonuniform lattices essentially coincides with Lubotzky's construction of uncountably many conjugacy classes of non-uniform lattices in $S L_{2}(k((t)))$ (see [18, p. 415]), where Conjecture 16.1 can be easily verified by dimension counting for $q \geq 4$, where $q=|k|$. Thus Conjecture 16.1 is true for $m=2$, and $q \geq 4$.

To give a geometric interpretation of the cuspidal deformations of $\Gamma$ in $G$ for $m=2$, and $q \geq 4$, we observe that the above discussion implies that we can vary the ends of $q$ of the $q+1$ cusps of $\Gamma$, each in an open neighborhood of the boundary of the Bruhat-Tits tree $X$. This gives a fundamental domain isomorphic to that of $\Gamma$, and isomorphisms of isotropy groups. 


\subsection{Conjugacy in Aut $(X)$}

Let $\mathbb{A}=(A, \mathcal{A})$ and $\mathbb{A}^{\prime}=\left(A^{\prime}, \mathcal{A}^{\prime}\right)$ be graphs of groups. We say that $\mathbb{A}$ and $\mathbb{A}^{\prime}$ are isomorphic, if there is an isomorphism

$$
\phi: A \longrightarrow A^{\prime}
$$

of graphs, and for each $v \in V A$, and each $e \in E A$, there are group isomorphisms

$$
\begin{aligned}
& \phi_{v}: \mathcal{A}_{v} \longrightarrow \mathcal{A}_{\phi(v)}^{\prime} \\
& \phi_{e}: \mathcal{A}_{e} \longrightarrow \mathcal{A}_{\phi(e)}^{\prime} .
\end{aligned}
$$

The cuspidal deformations of non-uniform lattices that we described above in Sec. 16.1 give rise to isomorphic graphs of groups. The following proposition indicates that the non-uniform lattices in the uncountably infinite families in Sec. 16.1 are all conjugate in $\operatorname{Aut}(X)$.

Proposition 16.1. Let $\Phi: \mathbb{A} \longrightarrow \mathbb{A}^{\prime}$ be an isomorphism of graphs of groups. Let $X=\widetilde{(\mathbb{A}, a)}$ and identify $X$ with $\left.\widetilde{\left(\mathbb{A}^{\prime}, a^{\prime}\right.}\right)$ via $\Phi$. Then $\pi_{1}(\mathbb{A}, a)$ and $\pi_{1}\left(\mathbb{A}^{\prime}, a^{\prime}\right)$ are conjugate in $\operatorname{Aut}(X)$.

Proof. The isomorphism $\Phi: \mathbb{A} \longrightarrow \mathbb{A}^{\prime}$ is a morphism of graphs of groups in the sense of $\left[1\right.$, Sec. 2]. Identifying $X=\widetilde{(\mathbb{A}, a)}$ with $\widetilde{\left(\mathbb{A}^{\prime}, a^{\prime}\right)}$ via $\Phi$, we get an induced automorphism of covering trees [1, Proposition 2.4]

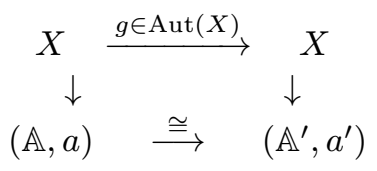

with the property that

$$
g \cdot \pi_{1}(\mathbb{A}, a)=\pi_{1}\left(\mathbb{A}^{\prime}, a^{\prime}\right) \cdot g
$$

that is, $\pi_{1}\left(\mathbb{A}^{\prime}, a^{\prime}\right)=g \pi_{1}(\mathbb{A}, a) g^{-1}$, for some $g \in \operatorname{Aut}(X)$.

Remark 16.1. If we take $\mathbb{A}$ and $\mathbb{A}^{\prime}$ to be graphs of trivial groups, then we obtain the result of Proposition 12.1.

\section{Failure of Property $T$ in Rank 2}

If a property $T$ group $H$ acts on a tree, then the group $H$ must fix a vertex [33]. Therefore, if a non-compact group $G$ acts on a tree with compact vertex stabilizers, $G$ cannot have property $T$. This is the case for the Kac-Moody group $G$ in the case $l=2$, and thus we have the following.

Proposition 17.1. When $l=2$, the group $G$ does not have Kazhdan's property $T$.

For a locally compact group $H$ containing lattices, $H$ has property $T$ if and only if lattices of $H$ have property $T$. Thus we obtain the following: 
Corollary 17.1. When $l=2$, lattices of $G$ do not have Kazhdan's property $T$.

Corollary 17.1 also follows from the fact that non-uniform lattices in $G$ cannot be finitely generated when $l=2$ (cf. Remark 15.1 (2)). Proposition 17.1 and Corollary 17.1 are in contrast to the higher rank case (see Sec. 18).

\section{Cohomology}

For $l \geq 3$, the methods of Garland in [12] yield results for various cohomologies on the Bruhat-Tits building $X$ associated with the $B N$-pair $(G, B, N)$, of our KacMoody group $G$, and on discrete subgroups $\Gamma \subseteq G$ (see also Ballmann-Swiatkowski [5], Pansu [24] and Zuk [35]). For example, we have the following:

Theorem 18.1. Let $\Gamma \subseteq G$ be a uniform lattice of $G$, and let

$$
\rho: \Gamma \longrightarrow \operatorname{Aut}(V)
$$

be a unitary representation $\Gamma$ in the complex Hilbert Space $V$ (not necessarily finite dimensional). If $l \geq 3$, and if $q=|k|$ is sufficiently large, then

$$
H^{i}(\Gamma, \rho)=0, \quad 0<i<l-1 .
$$

By Theorem 18.1 and [33] we have the following:

Corollary 18.1. Given $G$ and $q$ as in the theorem, if $G$ contains a uniform lattice, then $G$ has property $T$.

Applying the result of Valette and de la Harpe [33] in this way has already been utilized in the works of Ballmann-Swiatkowski, Pansu and Zuk cited above.

If $G$ is affine, then by Borel-Harder [4], we may deduce that $G$ contains uniform lattices when $l \geq 3$. However, if $G$ is not affine, we do not know if $G$ contains uniform lattices when $l \geq 3$.

Dymara and Januszkiewicz [10] have also used the results in [12], to obtain vanishing theorems, and to prove that $G$ has property $T$ when $l \geq 3$ and $G$ is of compact hyperbolic type. In [11] Dymara and Januszkiewicz give criteria for lattices in $G$ to have property $T$ in some non-compact cases.

It then follows (see, in contrast, Corollary 17.1) that in these cases the lattices we construct here (uniform and non-uniform) have property $T$. In particular these lattices are finitely generated and have finite commutator quotients. This is the case for the subgroups $\Gamma$ as in Theorem 8.1.

\section{Appendix. Explicit Computations for Symmetric, $2 \times 2$, Generalized Cartan Matrices}

We will consider matrices

$$
A=\left(\begin{array}{cc}
2 & -k \\
-k & 2
\end{array}\right), \quad k \in \mathbb{Z}_{\geq 3}
$$


We have

$$
\operatorname{det}(A-\lambda I)=\operatorname{det}\left(\begin{array}{cc}
2-\lambda & -k \\
-k & 2-\lambda
\end{array}\right)=\lambda^{2}-4 \lambda+\left(4-k^{2}\right) .
$$

Then the characteristic roots are given by:

$$
\lambda=2 \pm k .
$$

Indeed:

$$
\begin{gathered}
\left(\begin{array}{cc}
2 & -k \\
-k & 2
\end{array}\right)\left(\begin{array}{l}
1 \\
1
\end{array}\right)=\left(\begin{array}{l}
2-k \\
2-k
\end{array}\right)=(2-k)\left(\begin{array}{c}
1 \\
1
\end{array}\right) \\
\left(\begin{array}{cc}
2 & -k \\
-k & 2
\end{array}\right)\left(\begin{array}{c}
1 \\
-1
\end{array}\right)=\left(\begin{array}{c}
2+k \\
-(2+k)
\end{array}\right)=(2+k)\left(\begin{array}{c}
1 \\
-1
\end{array}\right),
\end{gathered}
$$

so

$$
f_{1}:=\left(\begin{array}{l}
1 \\
1
\end{array}\right), \quad f_{2}:=\left(\begin{array}{c}
1 \\
-1
\end{array}\right)
$$

are eigenvectors corresponding to eigenvalues $(2-k),(2+k)$, respectively. We have that

$$
e_{1}:=\left(\begin{array}{c}
1 / \sqrt{2} \\
1 / \sqrt{2}
\end{array}\right), \quad e_{2}:=\left(\begin{array}{c}
1 / \sqrt{2} \\
-1 / \sqrt{2}
\end{array}\right)
$$

constitute an orthonormal basis of eigenvectors. Relative to the basis $e_{1}, e_{2}$, the matrix $A$ then has the form

$$
A^{\prime}=\left(\begin{array}{cc}
2-k & 0 \\
0 & 2+k
\end{array}\right)
$$

and the corresponding quadratic form is

$$
\begin{aligned}
(x, y) A^{\prime}\left(\begin{array}{l}
x \\
y
\end{array}\right) & =x^{2}(2-k)+y^{2}(2+k) \\
& =(2-k)(2+k)\left(\frac{x^{2}}{2+k}-\frac{y^{2}}{k-2}\right), \quad k-2>0 .
\end{aligned}
$$

We consider the hyperbola

$$
\frac{x^{2}}{(2+k)}-\frac{y^{2}}{(k-2)}=2 .
$$

We recall that for a hyperbola

$$
\frac{x^{2}}{a^{2}}-\frac{y^{2}}{b^{2}}=c
$$

the asymptotes are given by

$$
\frac{x^{2}}{a^{2}}=\frac{y^{2}}{b^{2}}
$$


or

$$
y= \pm \frac{b}{a} x
$$

In our case,

$$
\begin{aligned}
& a^{2}=2+k \\
& b^{2}=k-2,
\end{aligned}
$$

and

$$
\frac{b^{2}}{a^{2}}=\frac{k-2}{k+2} \longrightarrow 1
$$

as $k \rightarrow \infty$. But also

$$
\frac{k-2}{k+2}<1 \text {. }
$$

So we have:

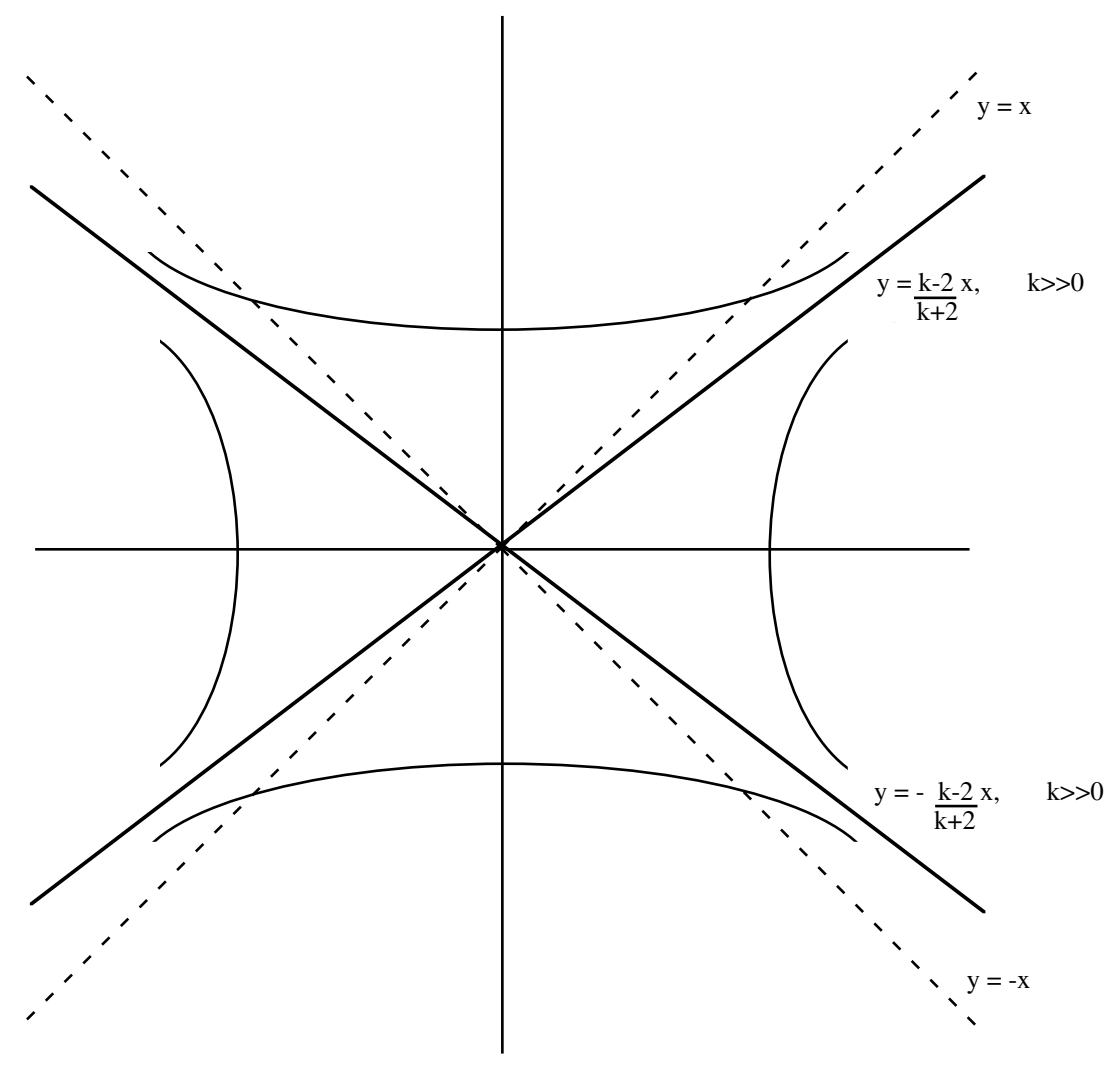

Fig. A.1. 
for the corresponding "system of hyperbolas". What are the asymptotes in the old coordinates? We have for the "old" canonical basis $f_{1}, f_{2}$

$$
\begin{aligned}
& e_{1}=\frac{1}{\sqrt{2}} f_{1}+\frac{1}{\sqrt{2}} f_{2} \\
& e_{2}=\frac{1}{\sqrt{2}} f_{1}-\frac{1}{\sqrt{2}} f_{2} .
\end{aligned}
$$

Hence

$$
\begin{aligned}
x e_{1}+y e_{2} & =\frac{1}{\sqrt{2}} x f_{1}+\frac{1}{\sqrt{2}} x f_{2}+\frac{1}{\sqrt{2}} y f_{1}-\frac{1}{\sqrt{2}} y f_{2} \\
& =\frac{1}{\sqrt{2}}(x+y) f_{1}+\frac{1}{\sqrt{2}}(x-y) f_{2},
\end{aligned}
$$

and the old coordinates $x^{\prime}, y^{\prime}$ (relative to $\left(f_{1}, f_{2}\right)$ ) are given in terms of the new coordinates $(x, y)$ (relative to $\left.\left(e_{1}, e_{2}\right)\right)$ by

$$
\begin{aligned}
& x^{\prime}=\frac{1}{\sqrt{2}}(x+y) \\
& y^{\prime}=\frac{1}{\sqrt{2}}(x-y) .
\end{aligned}
$$

The transform of the line

$$
y= \pm \frac{k-2}{k+2} x
$$

is

$$
\begin{aligned}
& x^{\prime}=\frac{1}{\sqrt{2}}\left(x \pm \frac{k-2}{k+2} x\right) \\
& y^{\prime}=\frac{1}{\sqrt{2}}\left(x \mp \frac{k-2}{k+2} x\right),
\end{aligned}
$$

or

$$
\begin{aligned}
& x^{\prime}=\frac{1}{\sqrt{2}}\left(\frac{(k+2) \pm(k-2)}{k+2} x\right) \\
& y^{\prime}=\frac{1}{\sqrt{2}}\left(\frac{(k+2) \mp(k-2)}{k+2} x\right) .
\end{aligned}
$$

This is the pair of lines:

$$
\begin{aligned}
& x^{\prime}=\frac{1}{\sqrt{2}}\left(\frac{2 k}{k+2} x\right) \\
& y^{\prime}=\frac{1}{\sqrt{2}}\left(\frac{4}{k+2} x\right),
\end{aligned}
$$


and

$$
\begin{aligned}
& x^{\prime}=\frac{1}{\sqrt{2}}\left(\frac{4}{k+2} x\right) \\
& y^{\prime}=\frac{1}{\sqrt{2}}\left(\frac{2 k}{k+2} x\right),
\end{aligned}
$$

or

$$
\frac{y^{\prime}}{x^{\prime}}=\frac{\frac{4}{k+2}}{\frac{2 k}{k+2}}=\frac{4}{2 k}=\frac{2}{k},
$$

and

$$
\frac{y^{\prime}}{x^{\prime}}=\frac{k}{2}
$$

Since $A$ is symmetric, the squared lengths of the simple roots $\alpha_{1}$ and $\alpha_{2}$ are the same and equal to 2, and hence all the Weyl roots are of equal length. The Weyl roots hence lie on the hyperbola $\frac{x^{2}}{(2+k)}-\frac{y^{2}}{(k-2)}=2$ with asymptotes $y^{\prime} / x^{\prime}=k / 2$ and $y^{\prime} / x^{\prime}=2 / k$ (see (14.9) and [20] for more details on hyperbolic root systems).

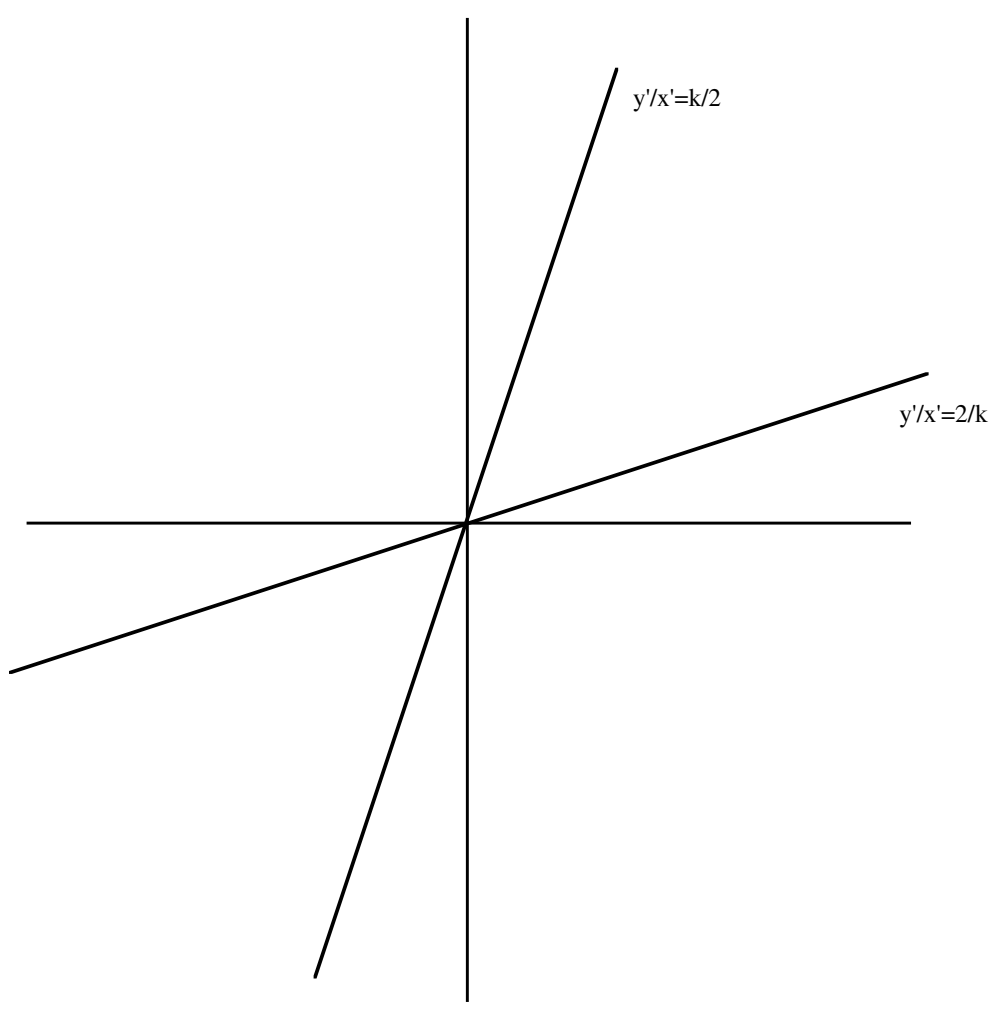

Fig. A.2. 


\section{Acknowledgment}

The first author was supported in part by NSF grant \#DMS-9800604. The authors would like to thank B. Remy and T. Januszkiewicz for their correspondence, and for informing us of their results. Thanks to A. Lubotzky for encouraging us to undertake this work and for explaining his constructions to us, and to H. Bass and I. Grojnowski for helpful discussions.

\section{References}

[1] H. Bass, Covering theory for graphs of groups, J. Pure Appl. Algebra 89 (1993) 3-47.

[2] N. Bourbaki, Groupes et algebres de Lie. Chapitres 4, 5 et 6, Elements de Mathematique, Masson, Paris, 1981.

[3] H. Bass and A. Lubotzky, Tree Lattices, Progress in Mathematics 176, Birkhauser, Boston 2000.

[4] A. Borel and G. Harder, Existence of discrete cocompact subgroups of reductive groups over local fields, J. Reine Angew. Math. 298 (1978) 53-64.

[5] W. Ballmann and J. Swiatkowski, On $L^{2}$-cohomology and property $(T)$ for automorphism groups of polyhedral cell complexes, Geom. Funct. Anal. 7 (1997) 615-645.

[6] L. Carbone, Deformations of lattices in rank 1 groups of $p$-adic type, preprint, 2000.

[7] L. Carbone and H. Garland, Lattices in Kac-Moody groups, Math. Research Letters 6 (1999) 439-447.

[8] D. Cartwright and M. Shapiro, Hyperbolic buildings, affine buildings and automatic groups, Michigan Math. J. 45 (1995) 511-523.

[9] W. Casselman and D. Wigner, Continuous cohomology and a conjecture of Serre's, Invent. Math. 25 (1974) 199-211.

[10] J. Dymara and T. Januszkiewicz, New Kazhdan groups, Geom. Dedicata 80 (2000) 311-317.

[11] J. Dymara and T. Januszkiewicz, Cohomology of buildings and of their automorphism groups, to appear in Invent. Math.

[12] H. Garland, p-adic curvature and the cohomology of discrete subgroups of $p$-adic groups, Ann. Math. 97 (1973) 375-423.

[13] H. Garland, The arithmetic theory of loop groups, Inst. Hautes tudes Sci. Publ. Math. 52 (1980) 5-136.

[14] H. Garland, A Cartan decomposition for p-adic loop groups, Math. Ann. 302 (1995) 151-175.

[15] H. Garland and J. Lepowsky, Lie algebra homology and the Macdonald-Kac formulas, Invent. Math. 34 (1976) 37-76.

[16] V. Kac, Infinite dimensional Lie algebras, Cambridge University Press, 1990.

[17] S. Kumar, Kac-Moody Groups, Their Flag Varieties and Representation Theory, Birkhauser, Boston Progress in Mathematics Series, 204, 2002.

[18] A. Lubotzky, Lattices in rank one Lie groups over local fields, Geom. Funct. Anal. 1 (1991) 405-431.

[19] Y. S. Liu, Density of the commensurability group of uniform tree lattices, J. Algebra 165 (1994) 346-359.

[20] J. Lepowsky and R. Moody, Hyperbolic Lie algebras and quasi-regular cusps on Hilbert modular surfaces, Math. Ann. 245 (1979) 63-88.

[21] J. Morita, Commutator relations in Kac-Moody groups, Proc. Japan. Acad., Series A 63 (1987) 21-22. 
[22] R. Moody and K. Teo, Tits systems with crystallographic Weyl groups, J. Algebra 21 (1972) 178-190.

[23] G. Moussong, Hyperbolic coxeter groups, PhD thesis, Ohio State University, 1988.

[24] P. Pansu, Formule de Matsushima, de Garland, et propriete $(T)$ pour des groupes agissant sur des espaces symetriques ou des immeubles, preprint, 1996.

[25] B. Remy, Formes presque deployees des groupes de Kac-Moody sur des corps quelconques, preprint, 1999.

[26] B. Remy, Immeubles a courbure negative et theorie de Kac-Moody, preprint, 1999.

[27] R. Steinberg, Lectures on Chevalley groups, Yale University, 1967.

[28] J.-P. Serre, Trees (Translated from the French by John Stilwell), Springer-Verlag, 1980.

[29] J. Tits, Resume de cours - theorie des groupes, Ann. College France (1980-1981), $75-87$.

[30] J. Tits, Uniqueness and presentation of Kac-Moody groups over fields, J. Algebra 105 (1987) $542-573$.

[31] J. Tits, Twin buildings and groups of Kac-Moody type, in LMS Lecture Notes 165 (Proceedings of a Conference on Groups, Combinatorics and Geometry, Durham 1990), Cambridge University Press, 1992, pp. 249-286.

[32] J. Tits, Sur le groupe des automorphisms d'un arbe, in Essays on Topology and Related Topics: Memories dedicated to George de Rham, Springer, 1970.

[33] A. Valette and P. de la Harpe, La propriete (T) de Kazhdan pour les groupes localement compacts, Asterisque 175 (1989).

[34] A. Weil, On discrete subgroups of Lie groups, Ann. Math. 72(2) (1960) 369-384.

[35] A. Zuk, La propriete $(T)$ de Kazhdan pour les groupes agissant sur des polyedres, C.R. Acad. Sci. Paris Serie I 323 (1996) 453-458. 